\title{
Estimación del balance de gases de efecto invernadero en sistemas de producción ganadera de la cuenca del río Salado
}

\author{
Jacobo, E. J., Cadaviz, N., Vecchio, M. C. y Rodriguez, A. M.
}

DOI: $10.31047 / 1668.298 x . v 37 . n 1.27514$

\begin{abstract}
RESUMEN
Se comparó el balance de gases efecto invernadero (GEI) de dos modelos de producción ganadera de la cuenca del río Salado, provincia de Buenos Aires. Uno, caracterizado por el uso predominante de pastizal natural en buena condición debido al pastoreo controlado (MP 1), y otro por una mayor superficie de pasturas y cultivos forrajeros, mayor carga animal y producción de carne (MP 2). Se estimaron las emisiones primarias según el IPCC (Panel Intergubernamental del Cambio Climático). Al balance de GEI se incorporaron las estimaciones de las emisiones secundarias y de la ganancia o pérdida de carbono del suelo. Las emisiones resultaron mayores en el MP 2 que en el MP 1 (4500 vs $2273 \mathrm{~kg} \mathrm{CO}$ eq. ha-1 año-1 respectivamente, $p<0,01$ ). El MP 1 secuestró carbono como carbono orgánico del suelo a una tasa de $1851 \mathrm{~kg}$

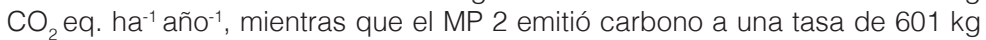
$\mathrm{CO}_{2}$ eq. ha-1 ${ }^{-1}$ añ-1. El balance de GEl fue diez veces más negativo en el MP 2 que en el MP 1, cuyo balance resultó neutro. Los sistemas pastoriles de esta región con predominio de pastizal natural bajo pastoreo controlado pueden mitigar los efectos del cambio climático.
\end{abstract}

Palabras clave: metano; secuestro de carbono; pastizal; método de pastoreo; agroecología.

Jacobo, E. J., Cadaviz, N., Vecchio, M. C. y Rodriguez, A. M. 2020. Estimation of the balance of greenhouse gases in livestock production systems in the Salado river basin. Agriscientia 37: 15-32

\section{SUMMARY}

We compared the balance of greenhouse gasses (GHG) of two contrasting livestock production models of the Salado river basin, Buenos Aires province. One model was characterized by the predominance of native grassland in good condition because of the application of controlled grazing (MP 1) and the other model by a larger surface of cultivated pastures and annual forage crops, higher stocking rate and beef production (MP 2). We applied the IPCC 
(Intergovernmental Panel on Climate Change) methodology for estimating primary emissions. We incorporated the estimation of secondary emissions and the gain or loss of soil carbon of to the GHG balance. Total emissions were higher in MP 2 than MP 1 (4500 vs. $2273 \mathrm{~kg} \mathrm{CO}_{2}$ eq. ha ${ }^{-1}$ year $^{-1}$ respectively, $\mathrm{p}<0,01)$. MP 1 sequestered carbon at a rate of $1851 \mathrm{~kg} \mathrm{CO}_{2}$ eq. $\mathrm{ha}^{-1}$ year ${ }^{-1}$, while MP 2 emitted carbon at a rate of $601 \mathrm{~kg} \mathrm{CO}_{2}$ eq. ha-1 year ${ }^{-1}$. GHF balance was ten times more negative for MP 2 than MP 1, whose balance resulted neutral. Livestock systems of Salado river basin with predominance of native grassland in good condition are able to mitigate the effect of global warming.

Keywords: methane; carbon sequestration; grassland; grazing method; agroecology.

Jacobo, E. J. (ORCID: 0000-0002-5622-7963), Cadaviz, N. (ORCID: 0000-0001-5671-7834), y Rodriguez, A. M. (ORCID: 0000-00023869-8518): Facultad de Agronomía, Universidad de Buenos Aires. Av. San Martin 4453, Ciudad Autónoma de Buenos Aires, Argentina, C1417DSE. Vecchio, M. C. (ORCID: 0000-0002-8284-2126): Facultad de Ciencias Agrarias y Forestales, Universidad Nacional de La Plata. Calle 60 y 119, La Plata, Buenos Aires, Argentina, B1900BVC. Correspondencia a: arodrigu@agro.uba.ar

\section{INTRODUCCIÓN}

En los últimos años se ha puesto en discusión el rol ambiental de la producción ganadera como causa de contaminación atmosférica con gases de efecto invernadero (GEI) (FAO, 2006). Las estimaciones de la contribución de fuentes directas del ganado al total de las emisiones antropogénicas globales varían entre 7,5 \% según U.S. EPA (2006) y 10,8 \% según O'Mara (2011). En Argentina, las emisiones provenientes de la producción agrícola y ganadera estimadas para el año 2012 alcanzaron el $27,8 \%$ de las emisiones totales de GEI del país. Este sector emitió el $95 \%$ del total del óxido nitroso $\left(\mathrm{N}_{2} \mathrm{O}\right)$, como consecuencia de las emisiones de los suelos agrícolas y el $59 \%$ del total de metano $\left(\mathrm{CH}_{4}\right)$, en su mayoría proveniente de la fermentación entérica (Secretaría de Ambiente y Desarrollo Sustentable, 2015). En el marco de esta discusión, una de las opciones planteadas para reducir la emisión de gases es la intensificación de la ganadería a través de la oferta de alimentos de mayor concentración energética (FAO y. New Zealand Agricultural Greenhouse Gas Research Centre, 2017).

El balance de GEI de los sistemas ganaderos es un flujo bidireccional que incluye, entre otros, el $\mathrm{CH}_{4}$ y el $\mathrm{N}_{2} \mathrm{O}$ emitido por el ganado y los suelos (flujo desde el sistema a la atmósfera) y la captura de $\mathrm{CO}_{2}$ por fotosíntesis secuestrada en los suelos (flujo desde la atmósfera al sistema). Sin embargo, los modelos que han sido desarrollados para estimar los balances de GEI de establecimientos ganaderos no suelen incluir posibles cambios en el stock de carbono de los suelos bajo distintos sistemas de manejo ganadero (Schils, Olesen, Del Prado y Soussana, 2007; Del Prado, Crosson, Olesen y Rotz, 2013). Tampoco se suelen incluir los cambios en el stock de carbono del suelo en los estudios que calculan la huella de carbono de los productos provenientes de sistemas pastoriles (Batalla et al. 2015).

Enlossistemasmás extensivos, donde laprincipal fuente de alimento es el forraje de pastoreo directo proveniente de pasturas cultivadas o pastizales naturales, el alimento tiene menor concentración de energía y nutrientes y mayor contenido de fibra que en los sistemas más intensivos, en los que se incrementa el aporte de granos y balanceados, de mayor concentración de energía y nutrientes (Fernández, 2010). La propuesta de intensificar la ganadería para reducir las emisiones de GEI se sostiene en el hecho de que los animales que consumen dietas con mayor proporción de concentrados y menor proporción de fibra generan una emisión de metano inferior (Lovett et al., 2005) y el período de tiempo requerido para completar el proceso de engorde también es más breve. 
Sin embargo, esto no implica necesariamente una menor emisión por hectárea, ya que estas están directamente relacionadas con la carga animal. Por otro lado, en áreas con predominio de pastizal natural, la intensificación de la ganadería requiere el reemplazo del pastizal por pasturas y cultivos forrajeros, lo que reduce la capacidad de secuestro de carbono de la vegetación (Soussana, Tallec y Blanfort, 2010). Además, en los sistemas más intensificados por mayor uso de insumos (semillas, maquinarias, combustibles, fertilizantes) las emisiones de otros gases, como $\mathrm{N}_{2} \mathrm{O}$ y $\mathrm{CO}_{2}$ son mayores por un uso más significativo de energía fósil y de fertilizantes (Nemecek, Dubois, HugueninElie y Gaillard, 2011). Aunque la discusión acerca de las estrategias productivas para mitigar las emisiones provenientes de la producción ganadera se ha generalizado en los últimos años, aún no hay consenso sobre las características del sistema de producción que libera menos GEI a la atmósfera (Cederberg y Stadig, 2003; Casey y Holden, 2006; Ogino, Orito, Shimada y Hirooka, 2007; Pelletier, Pirog y Rasmussen, 2010; Beauchemin, Janzen, Little, McAllister y McGinn, 2011). Hay estudios que apoyan la hipótesis de que la intensificación reduce la emisión de GEI (Pelletier et al. 2010), mientras que otros muestran que el aporte al calentamiento global de los sistemas pastoriles y/o de producción local de forrajes es menor que el de los intensificados (Cederberg y Stadig, 2003; Casey y Holden, 2006, Bellarby et al. 2013).

El secuestro de carbono realizado por pastizales y pasturas constituye un importante mecanismo de mitigación de las emisiones del sector ganadero (Soussana et al., 2010). El potencial de secuestro de carbono orgánico de los suelos de todos los pastizales y pasturas permanentes del mundo se ha estimado entre 0,01 y 0,3 giga toneladas de carbono al año (Lal, 2004), cuyo valor superior equivaldría al $4 \%$ del total de las emisiones mundiales (Soussana et al., 2010). El amplio rango de secuestro potencial de carbono de los sistemas pastoriles depende de la productividad de los recursos y de la naturaleza, frecuencia e intensidad de los disturbios asociados a los mismos. La capacidad de secuestro de carbono de los sistemas pastoriles aumenta cuando se incrementa la productividad de las pasturas y pastizales (Conant, Paustian y Elliot, 2001; Soussana et al., 2007). Otro factor que determina la capacidad de secuestro de carbono es el nivel de degradación de los sistemas pastoriles, tal como concluye un estudio que incluyó una importante cantidad de casos de sistemas pastoriles de varias regiones del mundo, donde se encontró que el stock de carbono promedio de los suelos de ecosistemas degradados fue $95 \%$ y $97 \%$ menor con respecto a valores de referencia, en sistemas templados y tropicales respectivamente (Ogle, Conant y Paustian, 2004). Este estudio también concluye que un factor a considerar en la capacidad de secuestro de carbono de los suelos es su nivel de saturación, ya que existe una relación inversa entre el contenido inicial de carbono de los suelos y su aumento debido a cambios en el manejo.

La Pampa inundable, con características ambientales que limitan severamente el uso agrícola, es un área de 9 millones de hectáreas ubicada al este de la provincia de Buenos Aires, cuya mayor superficie (6,5 millones de ha) está constituida por la cuenca baja del río Salado (Soriano et al., 1991). Esta región, que aún conserva entre el $53 \%$ y el $89 \%$ de pastizales naturales o seminaturales según la subregión (Vazquez y Rojas, 2006), es una de las principales zonas ganaderas del país ya que aloja alrededor del $13 \%$ del stock bovino nacional (Rearte, 2011a). Como resultado del proceso de expansión agrícola ocurrido en nuestro país en las últimas dos décadas, la ganadería ha sido expulsada de las zonas con mayor aptitud agrícola (Rearte, 2011b) y ha aumentado en áreas de suelos con limitantes como la cuenca del Salado. Por esta razón, la carga animal de la Pampa inundable se incrementó de 1,1 EV ha ${ }^{-1}$ en el año 2003 (Vázquez y Rojas, 2006) a 1,54 EVha-1 en el año 2007 (Vázquez, Rojas y Burgues, 2008). Las consecuencias de este proceso son: i) el deterioro del suelo y una baja estabilidad y productividad de los recursos forrajeros al reemplazar las comunidades naturales en ambientes con limitaciones (Jacobo y Rodríguez, 2009); ii) prácticas como la pulverización con glifosato sobre las comunidades más productivas, que alteraron su estacionalidad y redujeron drásticamente su riqueza, productividad primaria y el contenido de carbono de los suelos (Rodríguez y Jacobo, 2010; 2013; Rodríguez, Jacobo y Golluscio, 2018); iii) aumentos de carga por encima de su receptividad que deterioraron la vegetación natural (Jacobo y Rodríguez, 2009) y redujeron la productividad primaria neta (Recavarren y Martinefsky, 2009); y iv) aumento en el uso de insumos para la alimentación del ganado (Vázquez et al, 2008). Tanto la reducción de la superficie de pastizal natural como la degradación del pastizal remanente estarían afectando la capacidad de secuestro de carbono del pastizal de la región, sin embargo, no hay estudios que den cuenta de estos efectos. En la región, se ha documentado que la realización de un manejo adaptativo del pastizal (pastoreo controlado, según Deregibus, Jacobo y Rodríguez, 1995) mejora 
su condición ya que promueve grupos florísticos de alto valor forrajero y aumenta la cobertura del suelo (Jacobo, Rodríguez, Bartoloni y Deregibus, 2006). En este trabajo nos propusimos comparar el balance de gases de efecto invernadero de establecimientos ganaderos con dos modelos de producción diferentes: i) uno con bajo uso de insumos y utilización de una alta proporción de pastizal en buena condición como resultado del pastoreo controlado, y ii) otro, basado en elevado uso de insumos que utiliza baja proporción de pastizal en mala condición como resultado del pastoreo continuo. La estimación del balance de GEI se realizó utilizando la metodología propuesta por el IPCC (Panel Intergubernamental del Cambio Climático) y se utilizaron coeficientes provenientes de experimentos propios realizados en la zona para los cambios en el stock de Carbono de los suelos del pastizal.

\section{MATERIALES Y MÉTODOS}

\section{Sitio de estudio}

El estudio se realizó en la cuenca baja del río Salado, ubicada al sudeste de la Región Pampeana. El clima es templado húmedo, con un promedio anual de $1050 \mathrm{~mm}$ de precipitación y 16,5 ${ }^{\circ} \mathrm{C}$ de temperatura (período 1977-2009). Su relieve es casi plano: las pendientes no superan el $3 \%$ y presenta una predominancia de suelos salinos y/o alcalinos con drenaje deficiente, lo que determina la ocurrencia de anegamientos frecuentes (Soriano et al., 1991). Los establecimientos productivos estudiados (Figura 1) se seleccionaron de las dos subzonas más extensas de la cuenca del Salado y que conservan la mayor superficie de pastizal natural. Estas subzonas son la "ganadera de la costa" que incluye a los partidos lindantes con la bahía de Samborombón y la "ganadera de derrames", que incluye a los partidos ubicados en

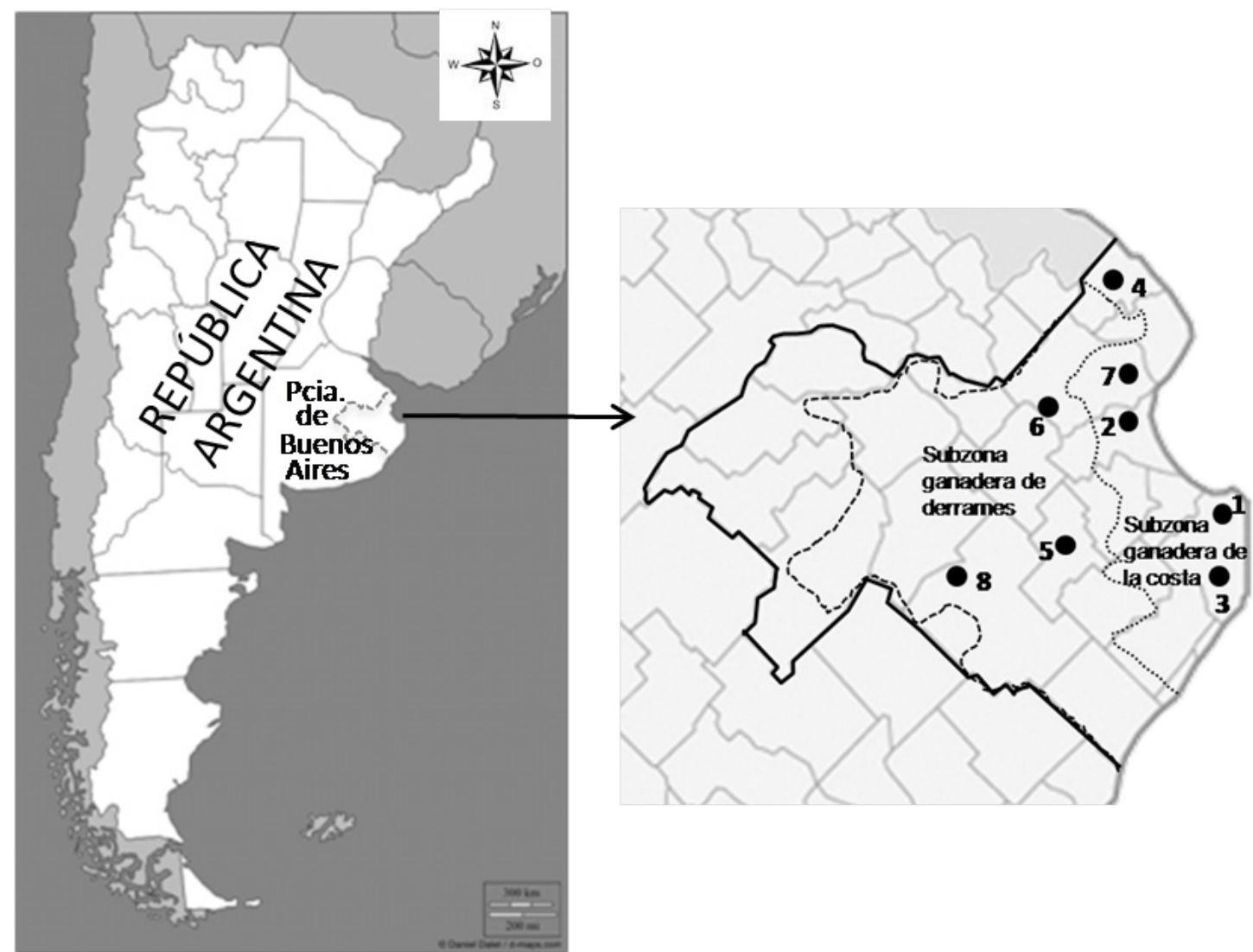

Figura 1. Ubicación de la cuenca del Salado en la República Argentina. A la derecha se indican los límites de las subzonas ganadera de la costa y ganadera de derrames, según Vazquez y Rojas (2006) y los ocho establecimientos que constituyeron los casos de estudio con un círculo negro y el número que los identifica. 
el centro de la cuenca del Salado, que conservaban el $89 \%$ y $86 \%$ de pastizales naturales con una relación vaca/total de animales de 0,48 y 0,50 respectivamente, según el censo 2002 (Vázquez y Rojas, 2006). La actividad tradicional de la región ha sido la cría de ganado bovino, aunque últimamente también se realiza recría e invernada.

\section{Casos analizados}

Se seleccionaron ocho casos (establecimientos ganaderos) que representaron dos modelos de producción diferentes: uno con bajo uso de insumos y utilización de una alta proporción de pastizal en buena condición como resultado del "pastoreo controlado" y otro basado en elevado uso de insumos para la alimentación del ganado y que utiliza baja proporción de pastizal en mala condición como resultado del pastoreo continuo (Tabla 1). Se reconoce como "pastoreo controlado" al pastoreo rotativo o intermitente diseñado sobre la base de conocimientos ecofisiológicos de la vegetación natural de los pastizales, con el fin de revertir el deterioro de la vegetación causado por el pastoreo continuo. Consiste en la aplicación de disturbios, principalmente pastoreos con altas cargas instantáneas y, eventualmente, desmalezadas o quemas, seguidos por descansos de duración variable. La intensidad y el momento de pastoreo, así como la duración del período de descanso, se determinan según el objetivo perseguido, tales como recuperar el vigor de las especies de alto valor forrajero, controlar la selectividad, modificar las relaciones de competencia entre grupos funcionales, promover la germinación y el establecimiento, así como la floración y fructificación de especies deseadas, (Deregibus et al., 1995). La aplicación del pastoreo controlado en los pastizales de la Pampa inundable incrementó la productividad primaria invernal (Jacobo, Rodríguez, Rossi, Salgado y Deregibus, 2000), la contribución relativa de los grupos funcionales de pastos y leguminosas de

Tabla 1. Descripción de los ocho establecimientos ganaderos analizados de la cuenca del Salado. En cada caso se indica el tipo de producción, la raza animal, la metodología de manejo del pastizal (C: Pastoreo controlado. NC: Pastoreo no controlado), la producción de carne, las características de los animales en pastoreo y en corral, la proporción de los distintos recursos forrajeros, la digestibilidad promedio de la dieta y la cantidad de nutrientes aportados por fertilización.

\begin{tabular}{|c|c|c|c|c|c|c|c|c|c|c|c|c|c|c|c|c|c|c|c|c|c|}
\hline \multirow[b]{2}{*}{$\begin{array}{l}\text { O } \\
\text { ர్ } \\
\text { U }\end{array}$} & \multirow[b]{2}{*}{$\begin{array}{l}\text { :0 } \\
\frac{0}{0} \\
\frac{0}{0} \\
\frac{7}{0} \\
\frac{0}{0} \\
0 \\
0 \\
0 \\
\stackrel{0}{=}\end{array}$} & \multirow[b]{2}{*}{ 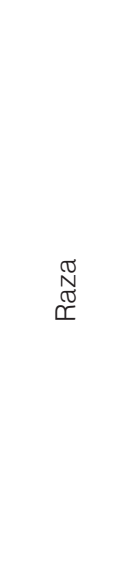 } & \multirow[b]{2}{*}{ 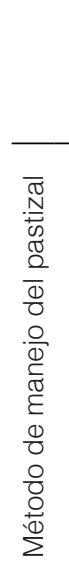 } & \multicolumn{5}{|c|}{$\begin{array}{c}\text { Animales en pastoreo } \\
\text { días/año) }\end{array}$} & \multicolumn{3}{|c|}{$\begin{array}{c}\text { Animales en } \\
\text { corral }\end{array}$} & \multicolumn{6}{|c|}{$\begin{array}{l}\text { Recursos forrajeros } \\
\text { Superficie relativa (\%) } \\
\text { o meses de uso }\end{array}$} & \multicolumn{2}{|c|}{$\begin{array}{l}\text { Digestibilidad } \\
\text { promedio (\%) }\end{array}$} & \multicolumn{2}{|c|}{$\begin{array}{l}\text { Aportes de } \\
\text { nutrientes } \\
\text { por } \\
\text { fertilización }\end{array}$} \\
\hline & & & & 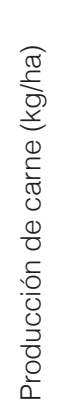 & 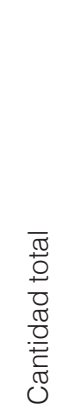 & 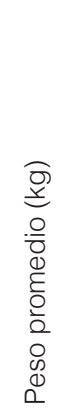 & 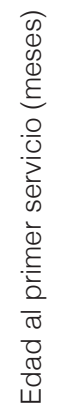 & 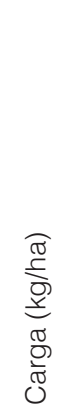 & 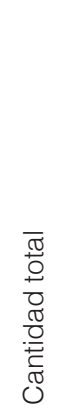 & 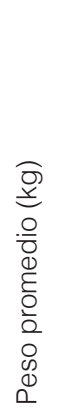 & 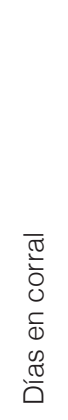 & 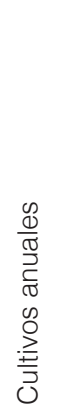 & 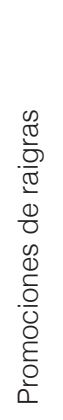 & 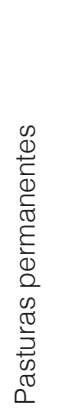 & 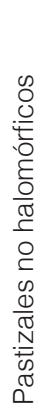 & 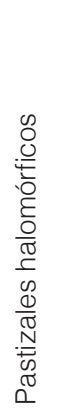 & 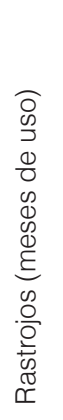 & 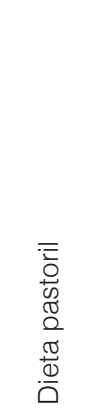 & 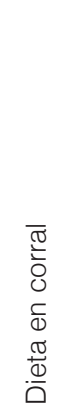 & 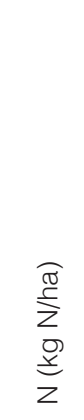 & 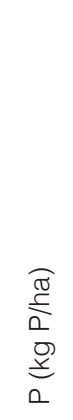 \\
\hline 1 & Cría & Angus & C & 99 & 1050 & 355 & 15 & 186 & 0 & & 0 & 0 & 0 & 0 & 85 & 15 & 0 & 64,1 & - & 0 & 0 \\
\hline 2 & $\begin{array}{l}\text { Ciclo } \\
\text { completo }\end{array}$ & Angus & C & 137 & 531 & 293 & 23 & 556 & 0 & & 0 & 7 & 0 & 0 & 93 & 0 & 2 & 62,0 & - & 0 & 0 \\
\hline 3 & Cría & Hereford & & 101 & 876 & 440 & 24 & 321 & 0 & & 0 & 0 & 0 & 0 & 83 & 17 & 0 & 63,5 & - & 0 & 0 \\
\hline 4 & Cría & Angus & C & 146 & 223 & 339 & 15 & 302 & 0 & & 0 & 4 & 8 & 4 & 77 & 6 & 0 & 65,6 & - & 5,5 & 6,5 \\
\hline 5 & $\begin{array}{l}\text { Cría/ } \\
\text { recría }\end{array}$ & Angus & C & 196 & 181 & 374 & 15 & 358 & 0 & & 0 & 13 & 0 & 0 & 87 & 0 & 5 & 58,2 & - & 3,2 & 3,7 \\
\hline 6 & $\begin{array}{l}\text { Ciclo } \\
\text { completo }\end{array}$ & Angus & NC: & 316 & 3423 & 313 & 15 & 456 & 1236 & & 158 & 10 & 12 & 29 & 0 & 49 & 0 & 60,1 & 80 & 10,7 & 29,2 \\
\hline 7 & $\begin{array}{l}\text { Ciclo } \\
\text { completo }\end{array}$ & Angus & NC: & 389 & 508 & 280 & 15 & 568 & 246 & 90 & 31 & 0 & 44 & 25 & 0 & 06 & 61,2 & 77 & 3 & 15,9 & \\
\hline 8 & Invernada & AAngus & NC: & 363 & 2089 & 257 & N/A & 686 & 0 & 0 & 21 & 34 & 28 & 14 & 2 & 06 & 62,4 & - & 25,6 & 13,8 & \\
\hline
\end{tabular}


mayor valor forrajero, y la receptividad del pastizal, sin afectar la diversidad florística, en comparación a los pastizales manejados bajo pastoreo continuo (Jacobo et al., 2006). De los ocho casos de estudio seleccionados, en los casos 1 a 5, la metodología de manejo del pastizal fue pastoreo controlado mientras que en los casos 6 a 8 el pastizal se manejó mediante pastoreo continuo. Las pasturas y cultivos forrajeros se manejaron mediante pastoreo rotativo en todos los casos. Con el fin de agrupar objetivamente los ocho establecimientos (casos) según los dos modelos de producción bajo estudio, se realizó un análisis de componentes principales (ACP)

En cada caso, las variables necesarias para estimar el balance de GEI se obtuvieron mediante encuestas semiestructuradas (Ander-Egg, 2001) y el relevamiento de los recursos forrajeros según la metodología descripta en Jacobo, Rodríguez, González y Golluscio (2016) para determinar la condición del pastizal. Para realizar el balance de GEI, en cada uno de los casos analizados se estimaron las emisiones de GEI y se sumó o restó (de acuerdo con el signo del cambio) el cambio estimado en el stock de carbono orgánico de los suelos (IPCC, 2006).

\section{Estimación de emisiones de GEI}

La emisión de GEl incluye las fuentes primarias, que son las producidas durante el proceso productivo y las fuentes secundarias, que ocurren durante la producción de los insumos utilizados durante el proceso productivo (Rotz, Montes y Chianese, 2010). La estimación de las emisiones primarias se realizó siguiendo la metodología acordada por el Panel Intergubernamental por el Cambio Climático, que asigna las emisiones secundarias a otros sectores de la economía (IPCC 2006). La metodología que incluye emisiones secundarias es la huella de carbono, que expresa el intercambio neto de todos los gases producidos por cada unidad de producto. En este trabajo se calcularon las emisiones secundarias según la metodología propuesta por Stackhouse-Lawson, Rotz, Oltjen y Mitloehner (2012) y se las expresó por hectárea para hacerlas comparables con las emisiones primarias.

Las fuentes primarias incluyen las emisiones resultantes de la gestión del ganado y del estiércol, tales como las emisiones de $\mathrm{CH}_{4}$ generadas por fermentación entérica y a partir de la deposición de estiércol; las emisiones de $\mathrm{N}_{2} \mathrm{O}$ a partir de las deposiciones de los animales en pastoreo y de los animales estabulados; las emisiones de $\mathrm{N}_{2} \mathrm{O}$ de los suelos gestionados y de $\mathrm{CO}_{2}$ derivadas de la aplicación de urea, de la descomposición de los restos vegetales aéreos y subterráneos, de la mineralización de la materia orgánica, de la deposición atmosférica y de la lixiviación y escurrimiento y de los cambios de carbono de los suelos. Para estimar las emisiones resultantes de la gestión del ganado y del estiércol se aplicó el nivel de resolución 2, ya que para cada caso (establecimiento) se registró o estimó la cantidad de animales de cada categoría, el peso vivo y el peso adulto, la ganancia diaria de peso, el tiempo de permanencia en pastoreo, el tiempo de terminación de las categorías de venta (Tabla 1), la digestibilidad y el contenido de proteína bruta de los alimentos (Tabla 2), la temperatura media invernal, etc. Estas variables se utilizaron para calcular los factores de emisión de las ecuaciones propuestas por el IPCC (2006) (Tabla 3). Para estimar las emisiones de $\mathrm{N}_{2} \mathrm{O}$ de los suelos gestionados y de $\mathrm{CO}_{2}$ derivadas de la aplicación de urea se utilizó el Nivel de resolución 1 por no disponer de factores de emisión específicos para las distintas

Tabla 2. Digestibilidad, Concentración energética y Proteína bruta de los los recursos forrajeros pastoriles, utilizados para calcular los factores de emisión de metano por fermentación entérica (EFfe) y de metano (EFge) y óxido nitroso $\left(\mathrm{EF}_{3}\right)$ por gestión del estiércol. El pastizal natural se separa según sean comunidades halomórficas (con salinidad y/o alcalinidad) o no halomórficas y según su condición.

\begin{tabular}{|c|c|c|c|c|}
\hline $\begin{array}{l}\text { Recurso } \\
\text { forrajero }\end{array}$ & $\begin{array}{l}\frac{\overline{0}}{0} \\
\frac{0}{0} \\
\frac{\check{c}}{0} \\
\circlearrowright\end{array}$ & $\begin{array}{l}\overline{0} \\
\frac{0}{0} \\
\frac{0}{\overline{0}} \\
\frac{0}{0} \\
0 \\
0 \\
0 \\
0 \\
0 \\
0\end{array}$ & 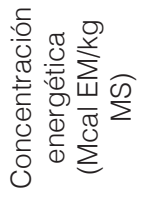 & 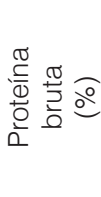 \\
\hline \multirow{2}{*}{$\begin{array}{l}\text { Pastizal no } \\
\text { halomórfico }\end{array}$} & Buena & 63,5 & 2,29 & 13 \\
\hline & Mala & 53 & 1,91 & 10 \\
\hline \multirow{2}{*}{$\begin{array}{l}\text { Pastizales } \\
\text { halomórfico }\end{array}$} & Buena & 55 & 1,98 & 10,5 \\
\hline & Mala & 43 & 1,55 & 8,5 \\
\hline $\begin{array}{l}\text { Promoción de } \\
\text { raigras }\end{array}$ & & 68 & 2,50 & 21,5 \\
\hline Pasturas & & 67 & 2,40 & 17 \\
\hline $\begin{array}{l}\text { Verdeos de } \\
\text { invierno }\end{array}$ & & 68 & 2,50 & 21,5 \\
\hline $\begin{array}{l}\text { Verdeos de } \\
\text { verano }\end{array}$ & & 66 & 2,40 & 13,5 \\
\hline $\begin{array}{l}\text { Rastrojos } \\
\text { de soja }\end{array}$ & & 41,7 & 1,5 & 4,8 \\
\hline
\end{tabular}

Fuentes consultadas: Hidalgo and Cahuépé (1991); Bavera, 2000; Fernández, 2010. 
Tabla 3. Ecuaciones y factores de emisión utilizados en las estimaciones de las emisiones primarias de los gases de efecto invernadero (GEI) según el proceso que genera la emisión (a) y del cambio de carbono de los suelos cultivados (b) según las directrices del IPCC 2006. Los niveles de resolución aplicados a cada proceso se indican al pie de la tabla.

\begin{tabular}{ll}
\hline a) EMISIONES PRIMARIAS & \\
\hline $\begin{array}{l}\text { Proceso que } \\
\text { genera la } \quad \text { GEI }\end{array}$ & Ecuaciones y factores de emisión
\end{tabular}

emisión

1. Emisiones por fermentación entérica $=\mathrm{EF}_{\mathrm{fe}}{ }^{*}\left(\right.$ cantidad total de animales $\left./ 10^{6}\right)$. EC. 10.19 $\mathrm{EF}_{\mathrm{fe}}$ (Factor de emisión de metano por fermentación entérica $)=\left(\mathrm{GE}^{\star}(\mathrm{Ym} / 100)\right.$ * días de permanencia) $/ 55,65$. Ec. 10.21

$\mathrm{GE}($ Energía bruta $)=(\mathrm{NEm}+\mathrm{NEa}+\mathrm{NEg}+\mathrm{NEI}+\mathrm{NEp}) / \mathrm{REM}) /$ (digestibilidad promedio de la dieta/100). EC. 10.16

Ym (Factor de conversión de metano). Cuadro 10.12

$\mathrm{NEm}$ (Energía neta para mantenimiento) $=\mathrm{Cfi}^{*}\left(\right.$ peso vivo $\left.{ }^{0,75}\right)$. EC. 10.3

Cfi es el coeficiente para calcular NEm función a la temperatura promedio de invierno. Ec 10.2 y Cuadro 10.4

NEa (Energía neta para actividad $)=\mathrm{Ca}$ * NEm. Ec 10.4

Ca es el coeficiente de actividad.Cuadro 10.5

$\mathrm{NEg}$ (Energía neta para crecimiento $)=22,02 *\left(\left(\text { peso vivo/ }\left(C^{*} \text { peso adulto }\right)\right)^{0,75}\right)$ * ganancia diaria de peso ${ }^{1,097}$. EC. 10.6

$\mathrm{C}$ es un coeficiente que toma valor 0.8 para hembras, 1.0 para machos castrados y 1.2 para toros (NRC, 1996)

NEI (Energía neta para leche en vacunos de carne) $=$ Cantidad de leche C $^{*}(1,47+0,4$ *

Metano contenido de grasa en la leche). Ec. 10.8

NEp (Energía neta de preñez) $=$ C preñez * NEm. Ec. 10.13

C preñez es el coeficiente de preñez. Cuadro 10.7

REM (Relación entre la energía neta disponible en el alimento y la energía digerible consumida $)=1,123-\left(4,092 * 10^{-3}\right.$ * digestibilidad promedio de la dieta $)+\left(12,126\right.$ * $10^{-5}$

Gestión del ganado y del estiércol

(i) * digestibilidad promedio de la dieta $\left.{ }^{2}\right)-(25,4 /$ digestibilidad promedio de la dieta). EC 10.14

2. Emisiones por gestión del estiércol $=\left(\mathrm{EF}_{\mathrm{ge}}{ }^{*}\right.$ cantidad total de animales $) / 10^{6}$. EC. 10.22

$\mathrm{EF}_{\mathrm{ge}}$ (Factor de emisión de metano por gestión del estiércol) $=(\mathrm{VS}$ * días de permanencia $)$ * $\left(\mathrm{B}_{0}{ }^{*} 0,67\right) *(\mathrm{MCF} / 100)$ * fracción de excreción total gestionado) Ec. 10.23

VS (Excreción de solidos volátiles en base a materia orgánica seca)= GE * (1 (digestibilidad promedio de la dieta/100) + UE) * ((1 - ceniza del estiércol como fracción de la ingesta de materia seca) / 18,45). Ec. 10.24:

UE (Energía urinaria como fracción de la energía bruta)=00.4*GE (IPCC 2006, pág. 10.46) Ceniza del estiércol como fracción de la ingesta de materia seca= 0.08 (IPCC 2006, pág. 10.46)

$\mathrm{B}_{0}$ (Capacidad máxima de producción de metano del estiércol). Cuadro 10A.5 MCF (Factor de conversión del metano). Cuadro 10.17

1. Emisión por excretas animales estabulados $=$ cantidad total de animales ${ }^{*}$ Nex * fracción de excreción total gestionado ${ }^{*} \mathrm{EF}_{3}{ }^{*}(44 / 28) . E C .10 .25$

Nex (Tasa de excreción anual de nitrógeno) $=(\mathrm{N}$ ingesta * $(1-$ fracción de la ingesta anual de nitrógeno retenida por el animal)) * días de permanencia. EC. 10.31

$\mathrm{N}$ ingesta (Ingesta anual de nitrógeno) $=(\mathrm{GE} / 18,45){ }^{*}(($ proteína bruta promedio de la

Óxido dieta/100) /6,25). EC. 10.32

nitroso $\mathrm{EF}_{3}$ (Factor de emisión para emisiones directas de óxido nitroso en el sistema de gestión del estiércol). Cuadro 10.21

2. Emisión por excretas animales en pastoreo $\left.=\mathrm{F}_{\mathrm{PRP}}{ }^{*} \mathrm{EF}_{3 \mathrm{PPP}, \mathrm{CPP}}\right)$ * $(44 / 28)$.EC. 11.1 $F_{\text {PRP }}$ (Cantidad anual de nitrógeno de la orina y el estiércol depositada por los animales en pastoreo $)=\left(\right.$ cantidad total de animales $\left.{ }^{*} \mathrm{Nex}\right){ }^{*}$ fracción de excreción anual total gestionado. EC. 11.5

$\mathrm{EF}_{3 \mathrm{PRP}, \mathrm{CPP}}($ Factor de emisión de óxido nitroso del $\mathrm{N}$ de la orina y el estiércol depositado en pastoreo). Cuadro 11.1 
1. Emisión por aplicación de fertilizantes $=F_{S N}$ (cantidad anual de nitrógeno aplicado en forma de fertilizantes sintéticos $\left.{ }^{\star} E F_{1}\right){ }^{\star}(44 / 28)$. EC. 11.1 (iii)

$\mathrm{EF}_{1}$ (Factor de emisión para emisiones de óxido nitroso de aportes de N). Cuadro 11.1

2. Emisión por aporte de nitrógeno en los residuos agrícolas $=\left(F_{C R}{ }^{*} E F_{1}\right) *(44 / 28)$. EC. 11.1 $\mathrm{F}_{\mathrm{CR}}$ (Cantidad anual de $\mathrm{N}$ en los residuos agrícolasaéreos y subterráneos)=rendimiento * superficie anual de cosecha* fracción del cultivo que se renueva anualmente * $\left(R_{A G}\right.$ * $N_{A G}{ }^{*}(1$ - fracción de los residuos aéreos que se extraen anualmente $\left.)+R_{B G}{ }^{*} N_{B G}\right)$. EC. 11.6 $R_{A G}$ (Relación entre la materia seca de los residuos aéreos y el rendimiento de cosecha): = $A_{\mathrm{DM}^{*}}$ * 1000 / rendimiento. IPCC 2006, pág. 11.15

$A G_{D M=}$ redimiento*declive+intercepción. Cuadro 11.2

$\mathrm{N}_{\mathrm{AG}}$ (Contenido de nitrógeno de los tejidos aéreos). Cuadro 11.2

$\mathrm{R}_{\mathrm{BG}}^{\mathrm{AG}}$ (Relación entre residuos subterráneos y rendimiento de cosecha $)=\mathrm{R}_{\mathrm{BG}-\mathrm{BIO}}{ }^{*}\left(\left(\mathrm{AG}_{\mathrm{DM}}{ }^{*} 1000\right.\right.$ + rendimiento)/rendimiento)

$\mathrm{R}_{\mathrm{BG}-\mathrm{BIO}}$ (Relación entre los residuos subterráneos y la biomasa aérea). Cuadro 11.2

$\mathrm{N}_{\mathrm{BG}}$ (Contenido de nitrógeno de los residuos subterráneos). Cuadro 11.2

Emisiones

de $\mathrm{N}_{2} \mathrm{O}$ de

los suelos

gestionados y

emisiones de

$\mathrm{CO}_{2}$ derivadas

de la aplicación

de cal y urea

(ii)
3. Emisión por cambio de materia orgánica del suelo $=\left(F_{\mathrm{SOM}}{ }^{*} E F_{1}\right){ }^{*}(44 / 28) . E C$. 11.1

$\mathrm{F}_{\text {som }}$ (Cantidad anual de $\mathrm{N}$ mineral que se mineraliza, relacionada con la perdida de $\mathrm{C}$ por cambios en el uso o la gestióndel suelo $)=\Delta \mathrm{C}$ mineral * $(1 / \mathrm{R}){ }^{*} 1000$. Ec.11.8

Óxido

nitroso

$\Delta \mathrm{C}$ mineral ${ }^{1}$ (Cambio anual en las existencias de carbono de los suelos minerales) $=($ contenido de referencia del C orgánico del suelo * factor de uso de la tierra ${ }^{2 *}$ factor de gestión * factor de entradas) - contenido de referencia del $\mathrm{C}$ orgánico del suelo Factor de uso de la tierra $\left(F_{L U}\right)$,Factor de gestión $\left(F_{M G}\right)$ y Factor de entradas $\left(F_{1}\right)$. Cuadros 5.5 y 6.2

R (Relación C:N de la materia orgánica del suelo) (IPPCC 2006, pág. 11.17)

$\mathrm{EF}_{1}$ (Factor de emisión para emisiones de óxido nitroso de aportes de N): Cuadro 11.1

4. Emisión de óxido nitroso producido por deposición atmosférica de nitrógeno volatilizado de suelos gestionados $=$ (cantidad anual de nitrógeno aplicado en forma de fertilizantes sintéticos $\left.\left.{ }^{\star} \mathrm{Frac}_{\mathrm{Gas}}\right)+\left(\mathrm{F}_{\mathrm{PRP}}{ }^{*} \mathrm{Frac}_{\mathrm{Gas}}\right){ }^{*} \mathrm{EF}_{4}\right){ }^{*}(44 / 28)$. EC. 11.9

$\mathrm{Frac}_{\mathrm{Gas}}$ (Fracción de $\mathrm{N}$ de fertilizantes sintéticos que se volatiliza como $\mathrm{NH}_{3}$ y $\mathrm{NO}_{\mathrm{x}}$ : Cuadro 11.3

$\mathrm{EF}_{4}$ (Factor de emisión correspondiente a las emisiones de óxido nitroso de la deposición atmosférica de N). Cuadro 11.3

5. Emisión de óxido nitroso por lixiviación/escurrimiento de nitrógeno de suelos gestionados $=($ cantidad anual de nitrógeno aplicado en forma de fertilizantes sintéticos $\left.\left.+\mathrm{F}_{\mathrm{PRP}}+\mathrm{F}_{\mathrm{CR}+} \mathrm{F}_{\mathrm{SOM}}\right){ }^{*} \mathrm{Frac}_{\text {Lixiviación }}{ }^{*} \mathrm{EF}_{5}\right){ }^{*}(44 / 28)$. EC. 11.10

Frac $_{\text {Lixivación }}$ (Fracción de todo el nitrógeno agregado/mineralizado en suelos gestionados). Cuadro 11.3

$\mathrm{EF}_{5}$ (Factor de emisión para emisión de óxido nitroso por lixiviación y escurrimiento). Cuadro 11.3

Dióxido 1. Emisión por aplicación de urea=(cantidad anual de fertilizante con Urea* FE) * (44/12).

de EC. 11.13

carbono FE (Factor de emisión): IPCC 2006, pág. 11.34

b) EMISIÓN O ABSORCIÓN DE CO ${ }_{2}$ POR CAMBIOS EN EL CONTENIDO DE CARBONO DE LOS SUELOS (iv)

1. $\Delta \mathrm{C}$ mineral(cambio anual de las existencias de $\mathrm{C}$ en suelos minerales, en ton año-1) $=\left(\mathrm{SOC}_{0}-\mathrm{SOC}_{(0-\mathrm{t})}\right) / \mathrm{DEC} .2 .25$

$\mathrm{SOC}=\sum\left(\mathrm{SOC}_{\mathrm{REF}}^{(0-t)}{ }^{*} \mathrm{~F}_{\mathrm{LU}}{ }^{*} \mathrm{~F}_{\mathrm{MG}}{ }^{*} \mathrm{~F}_{\mathrm{L}}{ }^{*}\right.$ superficie $)$

Cambio de $\mathrm{C}$ Dióxido $\quad \mathrm{SOC}_{\mathrm{REF}}$ : existencias de $\mathrm{C}$ de referencia. Cuadro 2.3

en los suelos carbono $\quad F_{L U}, F_{M G} y F_{L}$ son factores relativos al cambio de existencias durante 20 años para diferentes actividades de gestión de tierras. $F_{L U}$ factor de uso de la tierra, según tipo de cultivo; $\mathrm{F}_{\mathrm{MG}}$ factor de laboreo según tipo e intensidad y $\mathrm{F}_{\mathrm{L}}$ factor de entrada según la magnitud del retorno de los residuos de cosecha. Cuadro 5.5 .

(i) Para estas emisiones se utilizó el Nivel 2 (caracterización mejorada del ganado) dado que para cada caso (establecimiento) se registró o estimó la cantidad de animales de cada categoría, el peso vivo y el peso adulto, la ganancia diaria de peso, la cantidad de leche y el contenido de grasa de la leche, la digestibilidad y el contenido de proteína promedio de la dieta, los días de permanencia en pastoreo, la fracción de excreción anual total gestionado y la temperatura media invernal. Estas variables se destacan en negrita en las siguientes ecuaciones (Ec.), que se identifican en itálica con la numeración correspondiente según IPCC 2006. Se indica además la ecuación o tabla utilizada para obtener los coeficientes que integran las ecuaciones.

(ii) Para estas emisiones se utilizó el Nivel 1 por no disponer de factores de emisión específicos para las distintas condiciones bajo estudio (IPCC 2006, Cap. 11, pág.11.11). Las variables relevadas en cada caso (establecimiento) se destacan en negrita en las siguientes ecuaciones (Ec.), que se identifican en itálica con la numeración correspondiente según IPCC 2006. Se indica además la ecuación o tabla utilizada para obtener los coeficientes que integran las ecuaciones.

(iii) El único tipo de aporte de $\mathrm{N}$ que se aplicó en todos los casos fue como fertilizante sintético, por eso se omitieron los factores correspondientes a otros tipos de fertilizantes de la ecuación 11.1

(iv) Para los recursos forrajeros cultivados perennes y anuales se utilizó en Nivel 1 para estimar los cambios de C en el suelo (IPCC 2006, Cap. 2 y 5) 
condiciones bajo estudio. Las variables registradas o estimadas en cada caso (establecimiento), tales como superficie de cada tipo de cultivo utilizado para la alimentación animal, rendimiento y manejo agronómico (siembra, rotaciones, fertilización, etc.) fueron aplicadas en las ecuaciones o permitieron seleccionar el factor de emisión correspondiente, según lo propuesto por el IPCC (2006) (Tabla 3).

Las emisiones de $\mathrm{CH}_{4}$ y $\mathrm{N}_{2} \mathrm{O}$ estimadas se multiplicaron por sus coeficientes de conversión (25 y 298 respectivamente, IPCC 2006) para expresar los resultados en $\mathrm{CO}_{2}-\mathrm{C}$ equivalente $\left(\mathrm{CO}_{2}\right.$ eq.por unidad de superficie (ha) por año). Posteriormente, en cada caso se calculó el $\mathrm{CO}_{2}$ eq. por unidad de producto ( $\mathrm{kg}$ de peso vivo).

Las fuentes secundarias incluyen los gases $\mathrm{CO}_{2}$ y $\mathrm{N}_{2} \mathrm{O}$ emitidos en el proceso de producción de los insumos utilizados para la producción ganadera, tales como combustible, maquinaria, electricidad, fertilizantes, principios activos de los agroquímicos y todo el alimento comprado (granos, núcleo mineral, expeller de soja o girasol y urea). Para estimar las emisiones secundarias se multiplicó la cantidad utilizada de cada insumo por los factores de emisión de correspondiente, según la metodología propuesta por Stackhouse-Lawson et al. (2012).

\section{Estimación de cambios en el stock de carbono de los suelos}

El IPCC considera que el cambio en el stock de carbono en la biomasa y en la materia orgánica muerta es neutro para cultivos forrajeros herbáceos perennes (pasturas y pastizales) y anuales (verdeos). Por lo tanto, para estos recursos se estimó solamente el cambio en el stock de carbono en los suelos.

Para estimar los cambios de carbono en los suelos ocupados por recursos forrajeros cultivados perennes y anuales se utilizó el Nivel de resolución 1 por no disponer de factores de emisión específicos para las distintas condiciones bajo estudio. Estos cambios dependen de la gestión de los suelos según las prácticas agronómicas asociadas a estos cultivos forrajeros. Las variables registradas o estimadas en cada caso (establecimiento), tales como superficie de cada tipo de cultivo utilizado para la alimentación animal, rendimiento y manejo agronómico (siembra, rotaciones, fertilización, etc.) fueron aplicadas en las ecuaciones o permitieron seleccionar el factor de emisión correspondiente, según lo propuesto por el IPCC (2006) (Tabla 3).

Para estimar los cambios en el stock de carbono de los suelos de las comunidades de los pastizales bajo determinados manejos del pastoreo y prácticas agronómicas, se utilizaron coeficientes obtenidos de experimentos propios realizados en la zona de estudio. Para los pastizales de comunidades no halomórficas manejados bajo pastoreo controlado o sometidos a la aplicación recurrente de glifosato, práctica agronómica conocida como "promoción de raigras", los coeficientes se obtuvieron de Rodríguez, Jacobo y Golluscio (2018). El trabajo fue llevado a cabo en un establecimiento ganadero del centro de la cuenca del Salado (subzona ganadera de derrames), donde se tomaron muestras de suelo (Natracuol típico) de 0-12 cm de profundidad de tres lotes de comunidades no halomórficas manejados bajo pastoreo controlado y de otros tres lotes bajo la práctica de promoción de raigras, cada dos años entre los años 2008 y 2012. Los suelos de los pastizales bajo pastoreo controlado incrementaron el stock de carbono a una tasa de 0,539 $\mathrm{t} \mathrm{ha}^{-1} \mathrm{año}^{-1}$, mientras que los suelos bajo promoción de raigrás disminuyeron el stock de carbono a una tasa de 1,058 $\mathrm{t} \mathrm{ha}^{-1}$ año-1. Para los pastizales de comunidades halomórficas, los cambios en el stock de carbono del suelo como consecuencia de pastoreo controlado provinieron del experimento conducido por Vecchio, Golluscio, Rodríguez y Taboada (2018) en el este de la cuenca del Salado (subzona ganadera de la costa). Se tomaron muestras de 0-15 cm de cuatro lotes con suelos Natracualf vérticos, sometidos a pastoreo continuo o bajo pastoreo controlado durante los últimos nueve años para estimar el stock de carbono. Asumiendo que nueve años antes el stock de carbono de los suelos que actualmente están bajo pastoreo controlado era similar al de los suelos bajo pastoreo continuo, se estimó que el cambio de manejo de pastoreo continuo a controlado significó un aumento del stock de carbono a una tasa de 1,48 $\mathrm{t} \mathrm{ha}^{-1}$ año $\mathrm{o}^{-1}$.

Se calculó la variación de carbono del suelo de cada establecimiento como la sumatoria de la tasa de cambio de carbono de cada tipo de recurso forrajero multiplicado por la superficie que ocupa, en relación con la superficie total del establecimiento.

\section{Análisis de datos}

Para obtener un agrupamiento objetivo de los ocho establecimientos (casos) seleccionados según los dos modelos de producción bajo estudio, se realizó un análisis de componentes principales (ACP) utilizando la opción de matriz de coeficientes de correlación entre las variables que caracterizaron a cada caso: 1. Carga animal, 2. Producción de 
carne, 3. Superficie relativa de pastizal natural, 4. Superficie de pasturas y 5 . Superficie de cultivos forrajeros anuales y 6 . Condición del pastizal. Luego se aplicó el procedimiento MRPP (multiresponse permutation procedure) para confirmar el ordenamiento surgido del ACP e identificar grupos diferentes entre sí. Para ello se utilizó el programa estadístico PC ORD (Mccune y Mefford, 2006). Se compararon las diferencias entre los dos modelos productivos para las fuentes de emisión de GEI primarias y secundarias, la variación de carbono de los suelos y el balance de GEI mediante el test no paramétrico de Kolmogorov-Smirnov con el programa estadístico STATISTICA (StatSoft, Inc. 2007).

\section{RESULTADOSY DISCUSIÓN}

El análisis de componentes principales explicó el $94,7 \%$ de la variación total con los dos primeros componentes (CP 1 y CP 2). El CP 1, que explica el $87,27 \%$ de la variación total, discriminó a la derecha los casos 1 a 5, correspondientes a los sistemas de producción de carne bovina de la cuenca del Salado que hacen uso exclusivo o preponderante de pastizales naturales en buen estado de conservación como resultado del pastoreo controlado, utilizan una mínima proporción de forrajes cultivados y sustentan una menor carga animal (modelo productivo 1: MP 1), y a la izquierda, los casos 6 a 8 que corresponden a los sistemas de producción de carne que utilizan una mayor proporción de superficie de pasturas y cultivos forrajeros anuales, baja proporción de pastizal en mala condición como resultado del pastoreo continuo y sustentan una mayor carga

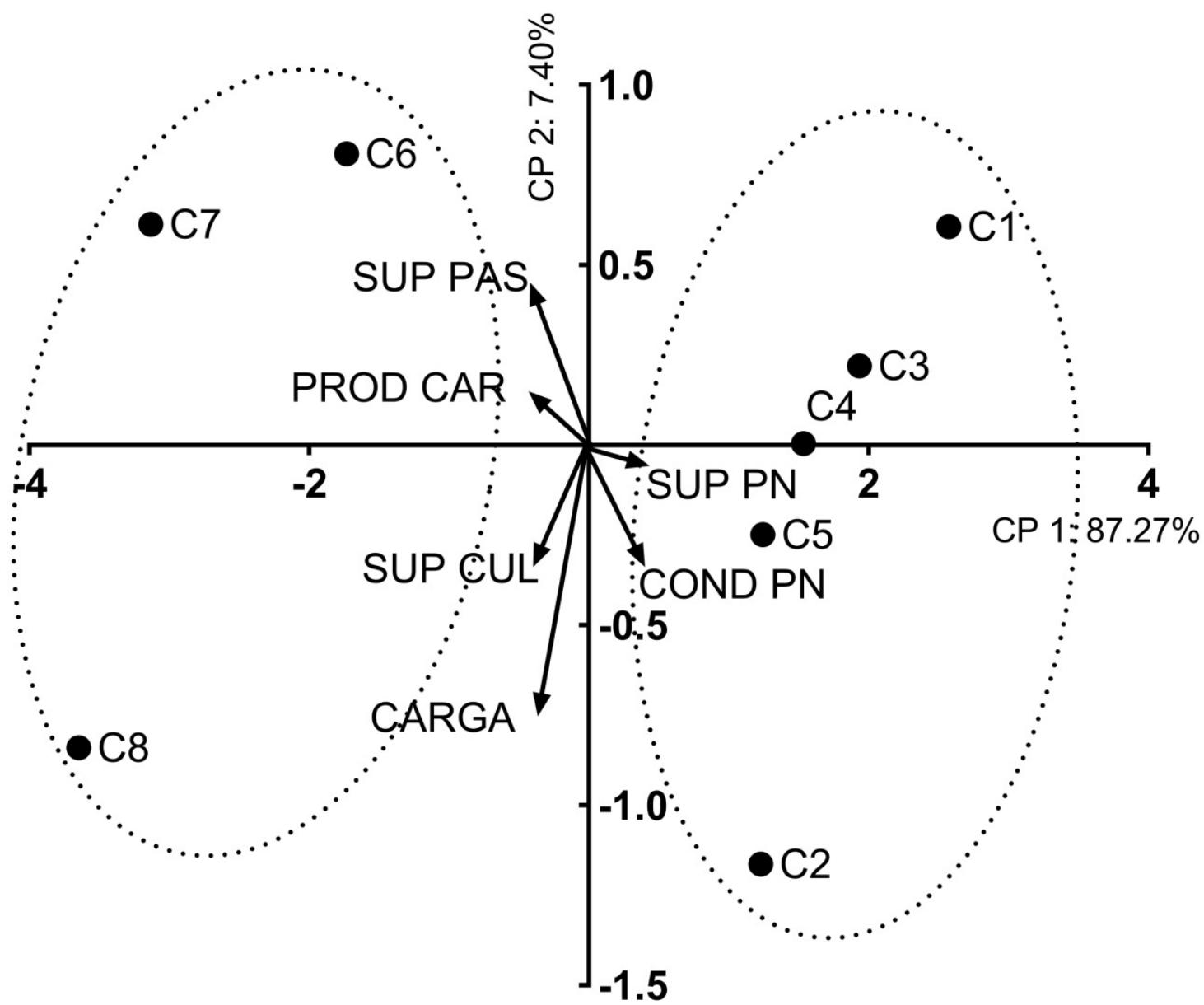

Figura 2. Ordenamiento de los establecimientos (casos, círculos negros) en relación con sus características (flechas) surgido del Análisis de Componentes Principales. SUP PN: Superficie relativa de pastizal natural, COND PN: Condición del pastizal natural, SUP PAS: Superficie relativa de pasturas, SUP CUL: Superficie de cultivos anuales, CARGA: Carga animal promedio, PROD CAR: Producción de carne. 
animal (modelo productivo 2: MP 2) (Figura 2). El procedimiento MRPP permitió confirmar la diferencia entre los modelos productivos ( $T=-$ 3,3349, $p=0,0108$ ).

Las emisiones primarias por unidad de superficie resultaron de una magnitud mucho mayor que las emisiones secundarias, alrededor de 150 veces en el MP 1 y 22 veces en el MP 2, mientras que ambas fueron superiores en el MP2 (Figura 3 a). Las emisiones primarias por unidad de carne producida no mostraron diferencias significativas entre modelos productivos, mientras que las emisiones secundarias por unidad de carne producida fueron superiores en el MP 2 (Figura 3 b). Las emisiones totales, que resultan de la suma de las emisiones primarias y secundarias, resultaron significativamente diferentes por unidad de superficie ( $4500 \pm 365$ vs. $2273 \pm 878 \mathrm{~kg} \mathrm{CO}_{2}$ eq. ha ${ }^{-1} a n ̃ o-1$, para el MP2 y MP1 respectivamente), mientras que las emisiones totales por unidad de producto animal no mostraron diferencias significativas $(12,66 \pm 0,30$ y 16,89 $\pm 5,14 \mathrm{~kg} \mathrm{CO}$ eq. $\mathrm{kg}$ vivo ${ }^{1}$, para el MP2 y MP1 respectivamente).

La mayor fuente de emisiones primarias fue el metano producto de la fermentación entérica, que constituyó entre el 69 y el 58 \% del total de estas emisiones en el MP 1 y en el MP 2 respectivamente (Tabla 4). La emisión de metano entérico por unidad de superficie del MP 2 tendió a ser superior a la del MP 1, mientras que la emisión de metano entérico por unidad de carne producida mostró una respuesta inversa, ya que la emisión del MP 2 tendió a ser inferior a la del MP 1 (Tabla 4 a).
Las emisiones de metano por unidad de superficie proveniente del estiércol, si bien resultaron de mucha menor magnitud que las del metano entérico, tendieron a ser superiores en el MP 2. Las distintas fuentes de emisiones primarias de óxido nitroso por unidad de superficie resultaron o tendieron a ser superiores en el MP 2 respecto del MP 1. Cuando las emisiones de óxido nitroso se expresaron por unidad de producto animal, sólo las provenientes del estiércol en corral, de la descomposición de los residuos de cosecha y de los cambios de la materia orgánica del suelo resultaron superiores en el MP 2 respecto del MP 1 (Tabla 4 a).

Respecto de las emisiones secundarias, todas las fuentes emitidas por el MP 2 resultaron superiores a las emitidas por el MP 1 cuando se expresaron por unidad de superficie. Cuando estas fuentes se expresaron por unidad de producto animal, las provenientes del uso de combustible, de fertilizantes fosforados, de productos fitosanitarios y del uso de alimentos fueron superiores en el MP 2 respecto del MP 1, mientras que las provenientes de la maquinaria y los fertilizantes nitrogenados no mostraron diferencias entre modelos productivos (Tabla 4 b).

Respecto de la variación de carbono del suelo, el MP 1 secuestró carbono a una tasa promedio

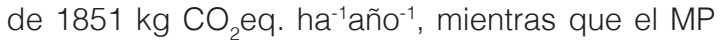
2 emitió carbono a una tasa promedio de $601 \mathrm{~kg}$ $\mathrm{CO}_{2}$ eq. ha-1 ${ }^{-1} \mathrm{ano}^{-1}$ (Figura 4). Como resultado de las diferencias entre las emisiones totales y el cambio de carbono en los suelos, el balance de gases a)

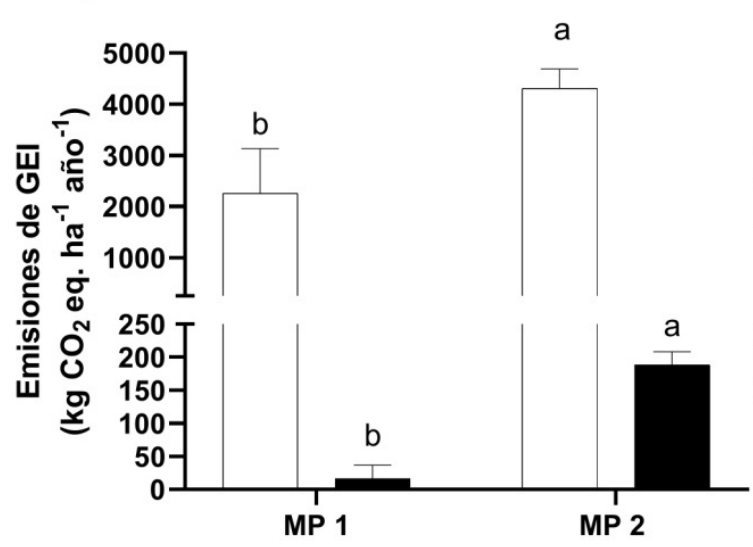

b)

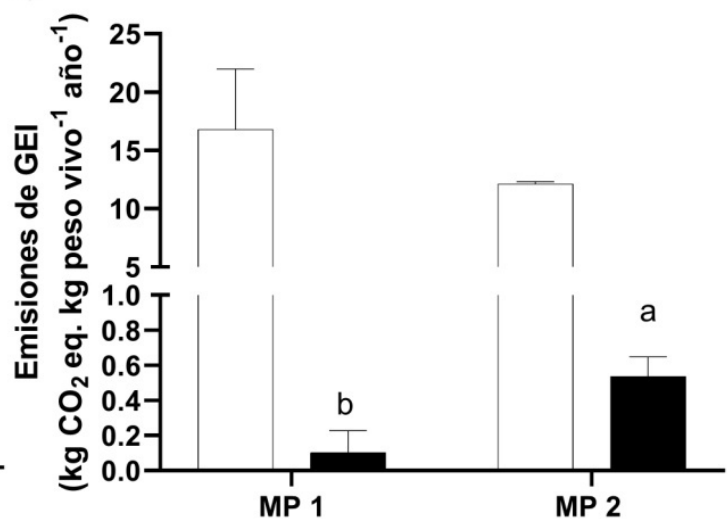

Figura 3. Emisión de gases de efecto invernadero proveniente de fuentes primarias (barras blancas) y secundarias (barras negras) a) por unidad de superficie (en kg CO2 eq. ha-1 año-1) y b) por unidad de producto (en kg CO2eq. kg de peso vivo-1 año-1) de dos modelos productivos ganaderos de la cuenca del Salado (MP 1: con alta proporción de pastizal natural en buen estado de conservación, y MP 2: con mayor proporción de cultivos forrajeros y carga animal). Letras distintas indican diferencias significativas entre modelos productivos para cada tipo de emisión $(p<0,05)$. 
Tabla 4. Comparación de las distintas fuentes de emisiones de GEl primarias (a) y secundarias (b) por unidad de superficie (kg CO 2 eq. ha $^{-1}$ año-1 y por unidad de producto (en $\mathrm{kg} \mathrm{CO}_{2}$ eq. $\mathrm{kg}^{-1}$ peso vivo ${ }^{-1}$ año- $^{-1}$ ) de los establecimientos ganaderos de la cuenca del Salado del MP 1 (con alta proporción de pastizal natural en buen estado) y del MP 2 (establecimientos con mayor proporción de cultivos forrajeros y carga animal), surgidos del test no paramétrico de Kolmogorov-Smirnov

\begin{tabular}{|c|c|c|c|c|c|c|c|c|c|c|}
\hline & \multicolumn{5}{|c|}{ Emisiones por unidad de superficie } & \multicolumn{5}{|c|}{ Emisiones por unidad de producto } \\
\hline & \multicolumn{2}{|c|}{ MP 1} & \multicolumn{2}{|c|}{ MP 2} & \multirow[t]{2}{*}{$\mathrm{p}$} & \multicolumn{2}{|c|}{ MP 1} & \multicolumn{2}{|c|}{ MP 2} & \multirow[t]{2}{*}{$\mathrm{p}$} \\
\hline & Media & $\mathrm{DE}$ & Media & $\mathrm{DE}$ & & Media & $\mathrm{DE}$ & Media & $\mathrm{DE}$ & \\
\hline \multicolumn{11}{|l|}{ a. Fuentes de emisiones primarias } \\
\hline $\mathrm{CH}_{4}$ entérico & 1555,18 & 633,93 & 2487,53 & 303,28 & 0,058 & 11,56 & 3,77 & 6,99 & 0,46 & 0,089 \\
\hline $\mathrm{CH}_{4}$ estiércol & 35,10 & 16,36 & 56,99 & 4,70 & 0,070 & 0,26 & 0,09 & 0,16 & 0,01 & 0,122 \\
\hline $\mathrm{NO}_{2}$ estiércol en pastoreo & 498,62 & 179,87 & 955,42 & 84,02 & 0,007 & 3,77 & 1,22 & 2,69 & 0,05 & 0,188 \\
\hline $\mathrm{NO}_{2}$ estiércol en corral & 0,00 & 0,00 & 63,15 & 58,60 & 0,043 & 0,00 & 0,00 & 0,18 & 0,16 & 0,037 \\
\hline \multirow{3}{*}{$\begin{array}{l}\mathrm{NO}_{2} \text { cambios } \mathrm{MO} \text { del suelo } \\
\mathrm{NO}_{2} \text { por lixiviación y } \\
\text { escurrimientodel suelo } \\
\mathrm{NO}_{2} \text { deposición atmosférica }\end{array}$} & 31,39 & 38,05 & 268,97 & 146,35 & 0,011 & 0,20 & 0,25 & 0,76 & 0,40 & 0,049 \\
\hline & 66,49 & 26,51 & 203,19 & 39,69 & 0,001 & 0,49 & 0,15 & 0,57 & 0,11 & 0,448 \\
\hline & 50,89 & 17,30 & 107,80 & 12,17 & 0,003 & 0,38 & 0,11 & 0,30 & 0,03 & 0,282 \\
\hline $\mathrm{NO}_{2}$ residuos cosecha & 9,69 & 10,91 & 95,11 & 37,73 & 0,003 & 0,07 & 0,08 & 0,26 & 0,09 & 0,016 \\
\hline $\mathrm{NO}_{2}$ fertilizantes & 8,14 & 11,73 & 61,29 & 53,86 & 0,067 & 0,05 & 0,08 & 0,17 & 0,15 & 0,162 \\
\hline $\mathrm{CO}_{2}$ por aplicación de urea & 1,34 & 2,29 & 12,33 & 18,99 & 0,225 & 0,01 & 0,02 & 0,03 & 0,05 & 0,324 \\
\hline Subtotal emisiones primarias & 2256,84 & 872,74 & 4311,78 & 380,86 & $<0,01$ & 16,79 & 5,18 & 12,13 & 0,20 & 0,182 \\
\hline \multicolumn{11}{|c|}{ b. Fuentes de emisiones secundarias } \\
\hline Combustibles & 1,53 & 1,48 & 19,69 & 0,75 & $<0,01$ & 0,01 & 0,01 & 0,06 & 0,01 & $<0,01$ \\
\hline Maquinaria & 1,50 & 3,14 & 10,07 & 3,94 & 0,014 & 0,01 & 0,02 & 0,03 & 0,01 & 0,110 \\
\hline Fertilizantes N & 5,75 & 8,29 & 43,32 & 38,07 & 0,067 & 0,04 & 0,05 & 0,12 & 0,10 & 0,162 \\
\hline Fertilizantes P & 2,09 & 3,04 & 20,19 & 8,59 & 0,004 & 0,01 & 0,02 & 0,06 & 0,03 & 0,044 \\
\hline \multirow{3}{*}{$\begin{array}{l}\text { Fitosanitarios } \\
\text { Granos para alimentación } \\
\text { animal } \\
\text { Otros alimentos }\end{array}$} & 4,85 & 5,38 & 37,16 & 9,78 & 0,001 & 0,03 & 0,03 & 0,11 & 0,04 & 0,024 \\
\hline & 0,78 & 0,98 & 4,70 & 1,96 & 0,008 & 0,00 & 0,01 & 0,01 & 0,00 & 0,065 \\
\hline & 0,00 & 0,00 & 53,07 & 47,04 & 0,037 & 0,00 & 0,00 & 0,15 & 0,13 & 0,034 \\
\hline Subtotal emisiones secundarias & 16,5 & 20.16 & 188,2 & 19.77 & $<0,01$ & 0,10 & 0,12 & 0,54 & 0,11 & 0,03 \\
\hline TOTAL EMISIONES & 2273,34 & 878,12 & 4499,98 & 364,84 & $<0,01$ & 16,89 & 5,14 & 12,66 & 0,30 & 0,21 \\
\hline
\end{tabular}

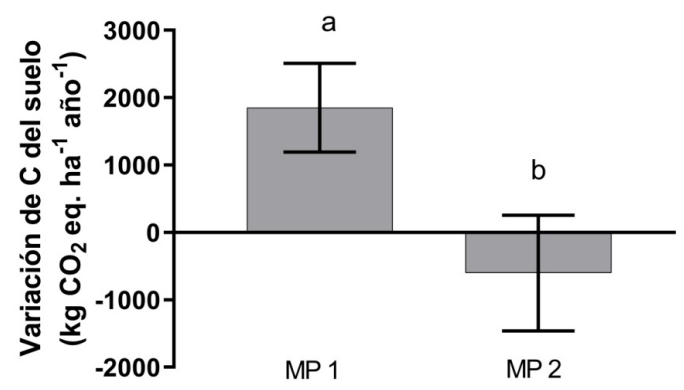

Figura 4. Cambio en el carbono orgánico de los suelos de los modelos productivos ganaderos de la cuenca del Salado (MP1: con alta proporción de pastizal natural en buen estado de conservación, y MP 2: con mayor proporción de cultivos forrajeros y carga animal). Valores positivos del eje indican secuestro y negativos emisión. Letras distintas indican diferencias significativas entre modelos productivos para cada tipo de emisión $(p<0,05)$.

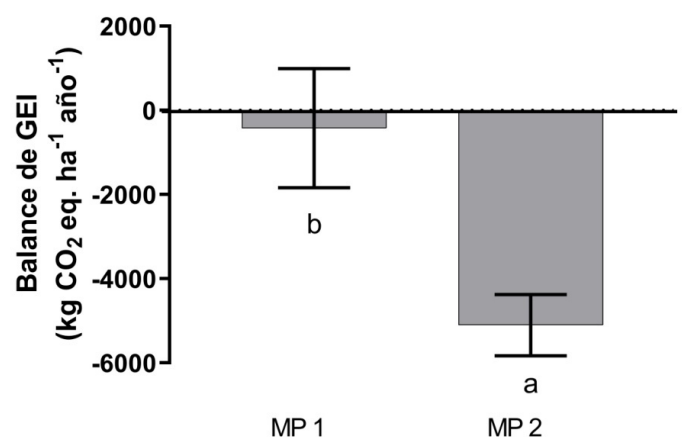

Figura 5. Balance de gases de efecto invernadero de los modelos productivos ganaderos de la cuenca del Salado (MP 1: con alta proporción de pastizal natural en buen estado de conservación y del MP 2: (con mayor proporción de cultivos forrajeros y carga animal). Letras distintas indican diferencias significativas entre modelos productivos para cada tipo de emisión $(p<0,05)$. 
efecto invernadero fue diez veces más negativo en el MP $2(-5101 \mathrm{~kg} \mathrm{CO} 2$ eq. ha-1 año-1) que en el MP 1 (-422 kg CO ${ }_{2}$ eq. ha ${ }^{-1}$ año-1) cuyo balance resultó prácticamente neutro (Figura 5).

Estos resultados sugieren que los sistemas pastoriles de producción de carne bovina más intensificados (MP 2), tienen un balance negativo de GEI, es decir, que son una fuente neta de gases. En cambio, los sistemas que utilizan menos insumos y realizan un adecuado manejo del pastoreo (MP 1) tienen un balance de GEI prácticamente neutro, es decir que están en equilibrio con la atmósfera. Estos resultados son consecuencia tanto de las mayores emisiones de GEI por unidad de superficie de los sistemas más intensificados, como de la mayor captación de carbono del suelo de los sistemas con base en el pastizal natural en buena condición como resultado del método de pastoreo.

El metano de origen entérico constituyó la principal fuente de emisiones generadas durante el proceso de producción de carne bovina en los dos modelos de producción de la cuenca del Salado, tal como se ha demostrado en diversos sistemas ganaderos (Schulze et al., 2009; Beauchemin, Janzen, Little, McAllister y McGinns, 2010). Así mismo, las emisiones de óxido nitroso provenientes del estiércol de los animales en pastoreo, también asociado a la carga animal, fueron la segunda fuente de emisiones primarias. En conjunto, las emisiones de metano y óxido nitroso por unidad de superficie fueron un $70 \%$ superior en el MP 2, cuya carga fue un $65 \%$ superior a la del MP 1. Nieto, Barrantes, Privitello y Reiné (2018) también encontraron que las menores emisiones por unidad de superficie se asocian con menores cargas en sistemas pastoriles semiáridos de Argentina. Debido a que las dos fuentes más importantes de GEI se asocian directamente con la carga, ésta se constituye en la variable determinante de las emisiones por unidad de superficie.

Por su parte, las emisiones de óxido nitroso y dióxido de carbono por unidad de superficie provenientes de las prácticas agrícolas, tales como siembra y fertilización de cultivos forrajeros representaron el 7 o el $17 \%$ de las emisiones totales del MP 1 y MP 2 respectivamente, siendo las del MP 2 tres veces y media superiores a las del MP 1. Estos resultados son consistentes con los hallados en otros sistemas extensivos, que asociaron las mayores emisiones de GEI a los establecimientos con mayor proporción de cultivos forrajeros anuales respecto de aquellos con mayor cobertura de pasturas perennes (Thomas, Sanderman, Eady, Masters y Sanford, 2012), o a la fertilización de los recursos forrajeros (Drewer et al., 2017). La mayor intensidad de prácticas agrícolas del MP 2 determinó que sus emisiones secundarias fueran superiores a las del MP 1, aunque en ambos casos fueron muy inferiores a las emisiones primarias. La magnitud de las emisiones de GEl provenientes de las prácticas agrícolas (tanto emisiones primarias como secundarias) resultó muy inferior a las generadas por la carga animal en ambos modelos productivos. Esto es consecuencia de que, entre los casos analizados, aun en los sistemas más intensificados, la mayor parte de las categorías realizan pastoreo directo el año completo, donde las pasturas perennes y aún el pastizal deteriorado, constituyen una fuente considerable de forraje. En sistemas pastoriles europeos también hallaron que la intensificación, resultante de duplicar la carga animal y fertilizar con nitrógeno mineral, incrementó las emisiones netas de GEl por unidad de superficie (Allard et al., 2007).

Si bien las emisiones de GEl por cada kilo de peso vivo no dan cuenta de la cantidad de gases de efecto invernadero liberados a la atmósfera por unidad de superficie, su cálculo es de interés ya que es un indicador de la eficiencia del proceso productivo. En este estudio las emisiones totales por unidad de producto animal alcanzaron un promedio de 12,66 y $16,89 \mathrm{~kg} \mathrm{CO}_{2}$ eq. $\mathrm{kg}$ vivo-1, para los MP 2 y MP1 respectivamente, y no fueron significativamente diferentes entre sí. Estos valores de eficiencia son similares a los calculados para los sistemas pastoriles de otras regiones, como Irlanda (13 kg CO 2 eq. kg vivo-1, Casey y Holden 2006), Canadá (11.88 kg CO ${ }_{2}$ eq. $\mathrm{kg}$ vivo $^{-1}$, Beauchemin et al. 2011) y Estados Unidos (12,7 y $13,5 \mathrm{~kg} \mathrm{CO}_{2}$ eq. $\mathrm{kg}$ vivo $^{-1}$, Stackhouse-Lawson et al., 2012). Se observo una alta variabilidad en el MP 1, debido a las elevadas emisiones de dos de los casos de este modelo. Por un lado, el establecimiento que realiza ciclo completo sobre pastizal natural emitió $25 \mathrm{~kg}$ $\mathrm{CO}_{2}$ eq. $\mathrm{kg}$ vivo $^{-1}$. La menor eficiencia registrada en este caso pone en evidencia que, si bien estos pastizales en buen estado de conservación son un recurso forrajero valioso, las bajas ganancias de peso que se alcanzan en las últimas etapas del engorde alargan este proceso y, en consecuencia, cuando se completa el ciclo productivo sobre pastizal natural se obtiene una baja producción de carne (137 kg de peso vivo ha-1 año-1). Además, esa baja producción se obtiene con una carga animal elevada (556 kg de peso vivo ha-1) debido a que en el campo coexisten distintas categorías, tales como vacas de cría, terneros en recría y animales en terminación. Por otro lado, el establecimiento que realiza cría de raza Hereford emitió $18,39 \mathrm{~kg}$ $\mathrm{CO}_{2}$ eq. $\mathrm{kg}$ vivo-1, $43 \%$ más que lo que emitieron 
los sistemas de cría de raza Angus, debido a que el peso de esos animales fue, en promedio, $85 \mathrm{~kg}$ superior a los de raza Angus y a que las vaquillonas requirieron más tiempo para entrar a servicio (24 meses vs 15 meses).

En lo que respecta a la contribución de la actividad ganadera al cambio climático, es bastante reciente considerar el secuestro de carbono de los suelos de pastizales y pasturas como un mecanismo de mitigación de las emisiones de GEl a la atmósfera (Soussana et al., 2004). La tasa a la que se produce el proceso de secuestro de carbono en los ambientes pastoriles depende de dos factores: el bioma y la naturaleza, frecuencia e intensidad de los disturbios a los que está sometido el sistema (Follett y Reed, 2010). En un meta-análisis que incluyó 115 estudios con 300 sitios de estudio, Conant et al. (2001) concluyeron que los biomas con mayor potencial de secuestro de carbono son los pastizales y las sabanas con un apropiado manejo del pastoreo. En este estudio, la tasa de secuestro de carbono estimada para los suelos de los establecimientos con predominio de pastizal natural en buenas condiciones de manejo (MP 1) resultaron en promedio $1851 \mathrm{~kg}$

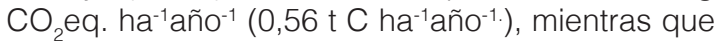
en los establecimientos en los que se reemplazó una importante proporción de pastizal por cultivos forrajeros o pasturas y además el pastizal remanente se manejó bajo pastoreo continuo (MP 2), los suelos no secuestraron carbono, sino que emitieron a una tasa de $601 \mathrm{~kg} \mathrm{CO}$ eq. ha-1 $a n ̃ o^{-1}\left(0,18\right.$ t $C$ ha $\left.^{-1} a n ̃ o-1\right)$. Los valores de secuestro de carbono calculados en este estudio para los establecimientos del MP 1 se encuentran dentro de los valores estimados o medidos en otros sistemas pastoriles del mundo. Los valores estimados de potencial de secuestro de carbono de los suelos de Norte América varían entre 0,2 y 0,56 t C ha-1 año-1, (Bruce et al., 1999; Conant et al.,2001), los de Australia entre 0,5 y 0,6 t C ha-1 año-1 (Gifford et al., 1992) y los valores medidos en diferentes climas y sistemas ganaderos de Europa son del orden de 1 t C ha-1 año-1 (Soussana et al., 2007).

Hay bastante consenso en que es insostenible que los pastizales actúen como un sumidero perpetuo de carbono debido a la saturación de carbono de sus suelos (Smith, 2014, Garnett et al. 2017). Estos autores postulan que los aumentos en el stock de carbono medidos en pastizales podrían reflejar un cambio pasado en el uso de la tierra (ej. de cultivo a pastizal) o una mejora en el sistema de manejo del pastoreo (ej. de continuo a rotativo). Sin embargo, las altas tasas de acumulación de carbono medidos en pastizales que nunca habían sido modificados ni cambiaron la gestión del pastoreo (Soussana et al. 2007), hace concluir a estos autores que sus resultados ponen en duda el concepto de saturación de carbono de los suelos.

El método de pastoreo, como regulador de los disturbios que soportan los sistemas pastoriles, es un factor importante en la determinación de la capacidad de secuestro de carbono del suelo (Conant, Cerri, Osborne y Paustian, 2017). Estos autores analizaron datos provenientes de 89 sistemas pastoriles diferentes y encontraron que la mejora en el método de pastoreo determinó incrementos en la tasa de secuestro de carbono del orden de las $0,28 \mathrm{t} \mathrm{C} \mathrm{ha-1} \mathrm{año}^{-1}$, aunque consideran que estos valores podrían estar subestimados porque los estudios analizados calculan el secuestro de carbono en horizontes superficiales. $\mathrm{Si}$ bien los efectos del manejo del pastizal se manifiestan principalmente en la superficie de los suelos, un estudio reciente muestra que $60 \%$ del carbono sensible al manejo del pastizal se acumula entre los 30 y $100 \mathrm{~cm}$ (Ward et al., 2016).

El cambio de carbono en el suelo generado por el pastoreo está relacionado con el clima, el tipo de suelo y la vegetación. (McSherry y Ritchie, 2013). Un modelo desarrollado por Meyer, Cullen y Eckard (2016) muestra que la capacidad de secuestro de carbono del suelo de un sistema pastoril depende de la concentración de carbono que caracteriza a cada tipo de suelo. Estos autores encuentran que el alto potencial de secuestro de los suelos con baja concentración de carbono, en algunos casos puede compensar las emisiones del sistema, y que la capacidad de compensación disminuye a medida que aumenta la materia orgánica de los suelos. En el mismo sentido, el potencial de secuestro de carbono es mucho mayor en suelos empobrecidos o degradados (Lal, 2003). La cuenca del Salado es una región con predominancia de suelos salinos y/o alcalinos con drenaje deficiente (Soriano et al., 1991) y un relativamente alto stock de carbono original (entre 54 y 71 t C ha $^{-1}$; Berhongaray, Alvarez, De Paepe, Caride y Cantet, 2013). Si bien las limitantes ambientales han restringido la expansión agrícola, una importante proporción de su superficie ha sido laboreada alguna vez y toda su superficie pastoreada de manera continua por más de un siglo, provocando su degradación (Deregibus, 1988) con la consecuente pérdida de carbono de sus suelos. En este sentido, las tasas de acumulación de carbono del suelo medidas en los experimentos realizados en la zona evidencian la alta potencialidad para secuestrar carbono en buenas condiciones de gestión del pastoreo. Asociado al contenido original de carbono de los suelos de las distintas comunidades, su capacidad de secuestro fue distinta. Mientras las comunidades 
de suelos halomórficos, con bajísimos niveles de carbono bajo pastoreo continuo, secuestraron a razón de1,48 t C ha-1 año-1 (Vecchio et al., 2018), las comunidades de suelos sin halomorfismo en superficie, con mayores contenidos de carbono, secuestraron a razón de 0,54 t C ha-1 año-1 (Rodríguez et al., 2018). Estas tasas son el resultado del cambio del contenido de carbono del suelo en los primeros cuatro años (comunidades no halomórficas) y nueve años (comunidades halomórficas) a partir de que se produjo el cambio en la metodología de pastoreo. Debido a que los cambios en el carbono del suelo basados en estudios a corto plazo tienen mayores tasas de secuestro que los de estudios de larga duración (Conant et al., 2017), podría esperarse que con el tiempo las tasas de acumulación se reduzcan. Sin embargo, se han documentado estudios de largo plazo que indican que los aumentos pueden continuar siendo lineales hasta los 40 años (Conant et al., 2001).

Como resultado de las menores emisiones de GEI por unidad de superficie y de la mayor fijación de carbono en los suelos de los establecimientos del MP 1, el balance de GEl se encuentra prácticamente en equilibrio con la atmósfera, mientras que el de los del MP 2 son una fuente neta de GEI. En sistemas pastoriles de Canadá también hallaron que, a carga baja, el balance de GEl fue prácticamente neutro mientras que, al duplicar la carga, se constituyó en fuente neta, emitiendo 37 veces más $\mathrm{CO}_{2}$ eq. ha-1 año-1 (McGinn, Beauchemin, Coates y McGeough, 2014). Asimismo, en sistemas pastoriles europeos encontraron que los casos cuya base forrajera fueron pasturas de corta duración o con un uso muy intensivo con corte y pastoreo, resultaron una fuente neta de GEI, a diferencia de los casos con predominio de pasturas perennes y pastoreo menos intensivo, que resultaron destino de GEI. (Soussana et al., 2007).

\section{CONCLUSIONES}

La metodología utilizada en este trabajo se sustenta en las estimaciones de emisiones y de cambios de carbono del suelo propuestas por el IPCC, a excepción de los cambios de carbono en el suelo de los pastizales bajo distintos manejos, en los que se utilizaron coeficientes generados a partir de datos de experimentos realizados en distintas comunidades del pastizal de la cuenca del Salado. Dado que la gestión del pastoreo es un factor relevante en la capacidad de secuestro de carbono de los pastizales, resulta crucial generar información para contar con coeficientes a nivel nacional, aplicables al Nivel 2 para la estimación del balance de GEI. En el mismo sentido, Viglizzo, Ricard, Taboada y Vázquez-Amábile (2019) advirtieron sobre la tendencia a subestimar el potencial de secuestro de carbono de los suelos en sistemas pastoriles al utilizar el procedimiento del IPCC. Estos autores demostraron que las ganancias de carbono en los ambientes pastoriles de Argentina, Uruguay, Paraguay y Brasil son superiores a las emisiones a cargas moderadas, y por lo tanto mitigan no sólo las emisiones del sector rural si no también, al menos parcialmente, las del sector no rural.

Estos resultados sugieren que los sistemas pastoriles de la cuenca del Salado, cuya base forrajera principal es el pastizal natural en buena condición como consecuencia de la metodología de pastoreo aplicada, pueden actuar como destinos importantes de carbono y, por lo tanto, mitigar los efectos del cambio climático.

\section{AGRADECIMIENTOS}

Las autoras agradecen muy especialmente a los productores y asesores que contestaron las encuestas y abrieron las puertas de sus establecimientos. Este trabajo se realizó con fondos provenientes de la Secretaría de Ciencia y Técnica de la Universidad de Buenos Aires (Proyectos UBACyT 01/Q339 y UBACyT 2018 538BA). Las autoras también agradecen a los revisores anónimos, cuyos comentarios mejoraron la calidad del trabajo.

\section{BIBLIOGRAFÍA}

Allard, V., Soussana, J.F., Falcimagne, R., Berbigier, P., Bonnefond, J.M., Ceschia E... Pinares-Patino C. (2007). The role of grazing management for the net biome productivity and greenhouse gas budget (CO2, $\mathrm{N} 2 \mathrm{O}$ and $\mathrm{CH} 4$ ) of semi-natural grassland. Agriculture,Ecosystems and Environment, 121 (1-2), 47-58. https://doi.org/10.1016/j.agee.2006.12.004

Ander-Egg, E. (2001). Métodos y técnicas de investigación social. Buenos Aires, Argentina: Grupo Editorial Lumen.

Batalla, I., Knudsen, M. T., Mogensen, L., del Hierro, Ó., Pinto, M. y Hermansen, J. E. (2015). Carbon footprint of milk from sheep farming systems in northern Spain including soil carbon sequestration in grasslands. Journal of Cleaner Production, 104, 121-129. https:// doi.org/10.1016/j.jclepro.2015.05.043 
Bavera, G. A. (2000). Digestibilidad de algunos forrajes empleados en bovinos en pastoreo. Recuperado de: https://documents.tips/documents/digestibilidadde-algunos-forrajes-empleados-en-empleados-enbovinos-a-pastoreo.html

Beauchemin, K. A., Janzen, H., Little, S., McAllister, T. y McGinn, S. (2010). Life cycle assessment of greenhouse gas emissions from beef production in western Canada: A case study. Agricultural Systems 103 (6), 371-379. https://doi.org/10.1016/j. agsy.2010.03.008

Beauchemin, K. A., Janzen, H., Little, S. McAllister, T. y McGinn, S. (2011). Mitigation of greenhouse gas emissions from beef production in western CanadaEvaluation using farm-based life cycle assessment. Animal Feed Science and Technology, 166-167, 663677. https://doi.org/10.1016/j.anifeedsci.2011.04.047

Bellarby, J., Tirado, R., Leip, A., Weiss, F., Lesschen, J. P. y Smith, P. (2013). Livestock greenhouse gas emissions and mitigation potential in Europe. Global change biology, 19 (1), 3-18. https://doi.org/10.1111/ j.1365-2486.2012.02786.x

Berhongaray, G., Alvarez, R., De Paepe, J., Caride, C. y Cantet, R. (2013). Land use effects on soil carbon in the Argentine Pampas. Geoderma, 192, 97-110. https://doi.org/10.1016/j.geoderma.2012.07.016

Bruce, J. P., Frome, M., Haites, E., Janzen, H., Lal, R. y Paustian, K. (1999). Carbon sequestration in soils. Journal of soil and water conservation, 54 (1), 382-389.

Casey, J. W. y Holden, N. M. (2006). Quantification of $\mathrm{GHG}$ emissions from sucker-beef production in Ireland. Agricultural Systems, 90 (1-3), 79-98. https:// doi.org/10.1016/j.agsy.2005.11.008

Cederberg, C. y Stadig, M. (2003). System expansion and allocation in life cycle assessment of milk and beef production. The International Journal of Life Cycle Assessment, 8 (6), 350-356. https://doi.org/10.1007/ BF02978508

Conant, R. T., Paustian, K. y Elliott E. T. (2001). Grassland management and conversion into grassland: effects on soil carbon. Ecological Applications 11 (2), 343-355. https://doi.org/10.1890/10510761(2001)011[0343:GMACIG]2.0.CO;2

Conant, R.T., Cerri, C.E., Osborne, B.B. y Paustian, K. (2017). Grassland management impacts on soil carbon stocks: a new synthesis. Ecological Applications 27(2), 662-668.https://doi.org/10.1002/eap.1473

Del Prado, A., Crosson, P., Olesen, J. E. y Rotz, C. (2013). Whole-farm models to quantify greenhouse gas emissions and their potential use for linking climate change mitigation and adaptation in temperate grassland ruminant-based farming systems. Animal, 7 (S2), 373-385. https://doi.org/10.1017/ S1751731113000748
Deregibus, V. A., Jacobo, E. J. y Rodríguez, A. M. (1995). Perspective: Improvement in rangeland condition of the Flooding Pampa of Argentina through controlled grazing. African Journal of Range \& Forage Science, 12 (2), 92-96. https://doi.org/10.1080/10220119.1995 .9647873

Deregibus, V. A. (1988). Importancia de los pastizales naturales en la República Argentina: situación presente y futura. Revista Argentina de Producción Animal, 8 (1), 67-78.

Drewer, J., Anderson, M., Levy, P. E., Scholtes, B., Helfter, C., Parker, J., ... Skiba U.M. (2017). The impact of ploughing intensively managed temperate grasslands on N2O, CH4 and CO2 fluxes. Plant Soil, 411,193-208. https://doi.org/10.1007/s11104-016-3023-x

FAO (Food and Agriculture Organization of the United Nations). (2006). Livestock's long shadow: environmental issues and options. Recuperado de: http://www.fao.org/docrep/010/a0701e/a0701e00. HTM.

FAO (Food and Agriculture Organization of the United Nations) y New Zealand Agricultural Greenhouse Gas Research Centre. (2017). Low-emissions development of the beef cattle sector in Argentina. Reducing enteric methane for food security and livelihoods. Recuperado de http://www.fao.org/3/a-i7671e.pdf

Fernández, H. (2010). Tablas de composición de alimentos para rumiantes. Recuperado de http:// www.produccion-animal.com.ar/tablas_composicion_ alimentos/46-Tabla.pdf

Follett, R. F. y Reed, D. A. (2010). Soil carbon sequestration in grazing lands: societal benefits and policy implications. Rangeland Ecology \& Management, 63 (1), 4-15. https://doi.org/10.2111/08-225.1

Garnett, T., Godde, C., Muller, A., Röös, E., Smith, P., de Boer, I.,...van Zanten, H. (2017). Grazed and confused? Food Climate Research Network, Environmental Change Institute, University of Oxford. Recuperado de https://www.fcrn.org.uk/projects/ grazed-and-confused

Gifford, R. M., Cheney, N. P., Noble, J. C., Russel, J. S., Wellington, A. B., Zammit, C. y Barson, M. M. (1992). Australian land use, primary production of vegetation and carbon pools in relation to atmospheric carbon dioxide concentration. En Gifford, R. M. y Barson, M. M. (Eds.), Australia's Renewable Resources: Sustainability and Global Change (pp. 151-187). Canberra, Australia: Australian Government Publishing Service.

Hidalgo, L. y Cahuépé, M. A. (1991). Producción de forraje de las comunidades de la Depresión del Salado. CREA, 149, 58-62.

IPCC (Intergovernmental Panel on Climate Change) (2006). Guidelines for National Greenhouse Gas 
Inventories. Recuperado de https://www.ipcc-nggip. iges.or.jp/public/2006gl/spanish/vol4.html

Jacobo, E. J. y Rodríguez, A. M. (2009). Valorización de pastizales naturales en ambientes húmedos. Indicadores de sustentabilidad. En Actas del V Congreso de la Asociación Argentina para el Manejo de los Pastizales Naturales (pp 20-29). Corrientes, Argentina: Asociación Argentina para el Manejo de los Pastizales Naturales.

Jacobo, E. J., Rodríguez, A. M. Bartoloni, N. y Deregibus, V. A. (2006). Rotational grazing effects on rangeland vegetation at a farm scale. Rangel andEcology \& Management, 59 (3), 249-257. https://doi. org/10.2111/05-129R1.1

Jacobo, E. J., Rodríguez, A. M., González, J. H. y Golluscio, R. A. (2016). Efectos de la intensificación ganadera sobre la eficiencia en el uso de la energía fósil y la conservación del pastizal en la cuenca baja del río Salado, Argentina. Agriscientia. 33 (1), 1-14. https://doi.org/10.31047/1668.298x.v33.n1.16567

Jacobo, E.J., Rodríguez, A. M., Rossi, J. L., Salgado, L., y Deregibus, V. A. (2000). Rotational stocking improves winter production of Italian ryegrass on argentinian rangelands. Journal of Range Management. 53 (5), 483-488. https://doi.org/10.2307/4003648

Lal, R. (2003). Global Potential of Soil Carbon Sequestration to Mitigate the Greenhouse Effect, Critical Reviews in Plant Sciences,22 (2),151-184. https://doi.org/10.1080/713610854

Lal, R. (2004). Soil carbon sequestration impacts on global climate change and food security. Science, 304 (5677), 1623-1627.https://doi.org/10.1126/ science. 1097396

Lovett, D., Stack, L., Lovell, S., Callan, J., Flynn, B., Hawkins, M. y O'Mara, F. (2005). Manipulating enteric methane emissions and animal performance of late-lactation dairy cows through concentrate supplementation at pasture. Journal of dairy science, 88 (8), 2836-2842. https://doi.org/10.3168/jds.S00220302(05)72964-7

Mccune, B., y Mefford, M. J. (2006). PC-ORD. Multivariate Analysis of Ecological Data. (Version 5.13)[Software]. Gleneden Beach, Oregon: MjM Software.

McGinn, S. M., Beauchemin, K. A., Coates, T. y McGeough, E. J. (2014). Cattle Methane Emission and Pasture Carbon Dioxide Balance of a Grazed Grassland. Journal of Environmental Quality, 43 (3), 820-828. https://doi.org/10.2134/jeq2013.09.0371.

McSherry, M. E. and Ritchie, M. E. (2013). Effects of grazing on grassland soil carbon: a global review. Global Change Biology, 19 (5),1347-1357. https://doi. org/10.1111/gcb.12144

Meyer, R., Cullen, B. R., y Eckard, R. J. (2016). Modelling the influence of soil carbon on net greenhouse gas emissions from grazed pastures. Animal Production Science, 56 (3), 585-593.https://doi.org/10.1071/ AN15508

Nemecek, T., Dubois, D., Huguenin-Elie, O. y Gaillard G. (2011). Life cycle assessment of Swiss farming systems: I. Integrated and organic farming. Agricultural Systems, 104 (3), 217-232. https://doi.org/10.1016/j. agsy.2010.10.002

Nieto, M. I., Barrantes, O., Privitello, L. y Reiné, R. (2018). Greenhouse Gas Emissions from Beef Grazing Systems in Semi-Arid Rangelands of Central Argentina. Sustainability, 10 (11), 4228. https://doi. org/10.3390/su10114228

O'Mara, F. P. (2011). The significance of livestock as a contributor to global greenhouse gas emissions today and inthe near future. Animal Feed Science and Technology,166-167, 7-15. https://doi.org/10.1016/j. anifeedsci.2011.04.074

Ogino, A., Orito, H., Shimada, K. y Hirooka, H. (2007). Evaluating environmental impacts of the Japanese beef cow-calf system by the life cycle assessment method. Animal Science Journal, 78 (4), 424-432. https://doi.org/10.1111/j.1740-0929.2007.00457.x

Ogle, S.M., Conant, R. T. y Paustian, K. (2004). Deriving grassland management factors for a carbon accounting method developed by the intergovernmental panel on climate change. Environmental Management, 33, 474484. https://doi.org/10.1007/s00267-003-9105-6

Pelletier, N., Pirog, R. y Rasmussen, R. (2010). Comparative life cycle environmental impacts of three beef production strategies in the Upper Midwestern United States. Agricultural Systems, 103 (6), 380-389. https://doi.org/10.1016/j.agsy.2010.03.009

Rearte, D. (2011a). Situación actual y prospectiva de la situación de carne vacuna. INTA. Recuperado de: https://inta.gob.ar/documentos/situacion-actual-yprospectiva-de-la-produccion-de-carne-vacuna

Rearte, D. (2011b). Situación actual y prospectiva de la ganadería argentina, un enfoque regional. Archivos Latinoamericanos de Producción Animal, 19 (3-4), 4649.

Recavarren, P. M. y Martinefsky, M. J. (2009). Alerta amarillo: la degradación de los recursos forrajeros. Visión Rural, 16 (77), 29-31.

Rodríguez, A. M. y Jacobo E. J. (2010). Glyphosate effects on floristic composition and species diversity in the Flooding Pampa grassland (Argentina). Agriculture, Ecosystems \& Environment, 138 (3-4), 222-231. https://doi.org/10.1016/j.agee.2010.05.003

Rodríguez, A. M. y Jacobo, E. J. (2013). Glyphosate effects on seed bank andvegetation composition of temperate grasslands. Applied Vegetation Science, 16 (1), 51-62. https://doi.org/10.1111/j.1654109X.2012.01213.x 
Rodríguez, A. M., Jacobo, E. J. y Golluscio, R. A. (2018). Glyphosate Alters Aboveground Net Primary Production, Soil Organic Carbon and Nutrients in Pampean Grasslands (Argentina). Rangeland Ecology \& Management, 71(1), 119-125. https://doi. org/10.1016/j.rama.2017.07.009

Rotz, C. A., Montes, F. y Chianese, D. S. (2010). The carbon footprint of dairy production systems through partial life cycle assessment. Journal of Dairy Science, 93 (3), 1266-1282. https://doi.org/10.3168/jds.20092162

Schils, R., Olesen, J. E., Del Prado, A. y Soussana, J. (2007). A review of farm level modelling approaches for mitigating greenhouse gas emissions from ruminant livestock systems. Livestock Science, 112 (3), 240251. https://doi.org/10.1016/j.livsci.2007.09.005

Schulze, E., Luyssaert, S., Ciais, P., Freibauer, A., Janssens, I. A., Soussana, J. F,...the CarboEurope team. (2009). Importance of methane and nitrous oxide for Europe's terrestrial greenhouse-gas balance. NatureGeoscience, 2, 842-850. https://doi. org/10.1038/ngeo686

Secretaría de Ambiente y Desarrollo Sustentable (2015). $3^{\text {a }}$ Comunicación Nacional de la República Argentina a la Convención Marco de las Naciones Unidas sobre Cambio Climático. Recuperado de https://unfccc.int/ sites/default/files/resource/Argnc3.pdf

Smith, P. (2014). Do grasslands act as a perpetual sink for carbon? Global Change Biology, 20 (9), 2708-2711. https://doi.org/10.1111/gcb.12561

Soriano, A., León, R. J. C., Sala, O. E., Lavado, R. S., Deregibus, V. A., Cauhépé, M. A., ... y Lemcoff, J. H. (1991). Río de la Plata grasslands. En Couplan, R. T. (ed.), Natural grasslands: introduction and Western Hemisphere (367-407). Amsterdam, Netherlands: Elsevier.

Soussana, J. T., Loiseau, P., Vuichard, N. Ceschia, E., Balesdent , J. Chevallier,T. y Arrouays, D. (2004). Carbon cycling and sequestration opportunities in temperate grasslands. Soil Use and Management, 20 (2), 219-230.https://doi.org/10.1111/j.1475-2743.2004. tb00362.x

Soussana, J., Allard, V., Pilegaard K., Ambus, P., Amman, C., Campbell, C., ...Valentini, R. (2007). Full accounting of the greenhouse gas (CO2, N2O, CH4) budget of nine European grassland sites. Agriculture,
Ecosystems \& Environment, 121 (1-2), 121-134. https://doi.org/10.1016/j.agee.2006.12.022

Soussana, J., Tallec, T. y Blanfort, V. (2010). Mitigating the greenhouse gas balance of ruminant production systems through carbon sequestration in grasslands. Animal, 4 (3), 334-350. https://doi.org/10.1017/ S1751731109990784

Stackhouse-Lawson, K., Rotz, C., Oltjen, J. y Mitloehner, F. (2012). Carbon footprint and ammonia emissions of California beef production systems. Journal of Animal Science,90(12), 4641-4655. https://doi.org/10.2527/ jas.2011-4653

StatSoft, Inc. (2007). STATISTICA (version 8.0) [Software] www.statsoft.com.

Thomas, D.T., Sanderman, J., Eady, S.J., Masters, D.G.y Sanford, P. (2012). Whole Farm Net Greenhouse Gas Abatement from Establishing Kikuyu-Based Perennial Pastures in South-Western Australia. Animals, 2 (3), 316-330. https://doi.org/10.3390/ani2030316

United States Environmental Protection Agency (U.S. EPA). (2006). Global anthropogenic mon-CO2 greenhouse gas emissions: 1990-2020.

Vázquez, P. y Rojas, M. (2006). Zonificación Agroecológica del área de Influencia de la EEA Cuenca del Salado. (Publicación Técnica Nº 2). Buenos Aires, Argentina: Ediciones INTA.

Vázquez, P., Rojas, M. y Burges, J. (2008). Caracterización y tendencias de la ganadería bovina en la cuenca del Salado. Veterinaria Argentina 25 (248), 572-584.

Vecchio, M. C., Golluscio, R. A., Rodriguez, A. M. y Taboada, M. A. (2018). Improvement of Saline-Sodic Grassland Soils Properties by Rotational Grazing in Argentina. Rangeland Ecology \& Management, 71 (6), 807- 814. https://doi.org/10.1016/j.rama.2018.04.010

Viglizzo, E. F., Ricard, M. F., Taboada, M. A. y VázquezAmábile, G. (2019). Reassessing the role of grazing lands in carbon-balance estimations: Meta-analysis and review. Science of the Total Environment, 661, 531542. https://doi.org/10.1016/j.scitotenv.2019.01.130

Ward, S. E., Smart, S. M., Quirk, H., Tallowin, J. R., Mortimer, S. R., Shiel, R. S., y Bardgett, R. D. (2016). Legacy effects of grassland management on soil carbon to depth. Global change biology, 22 (8), 29292938. https://doi.org/10.1111/gcb.13246 